\title{
Radar Location Equipment Development Program Phase I
}
G. A. Sandness
K. C. Davis

June 1985

Prepared for the

Westinghouse Electric Corporation under a Related Services Agreement with the U.S. Department of Energy Contract DE-AC06-76RLO 1830

Pacific Northwest Laboratory Operated for the U.S. Department of Energy by Battelle Memorial Institute 


\title{
DISCLAIMER
}

This report was prepared as an account of work sponsored by an agency of the United States Government. Neither the United States Covernment nor any agency thereof, nor any of their employees, makes any warranty, express or implied, or assumes any legal liability or responsibility for the accuracy, completeness, or usefulness of any information, apparatus, product, or process disclosed, or represents that its use would not infringe privately owned rights. Reference herein to any specific commercial product, process, or service by trade name, trademark, manufacturer, or otherwise, does not necessarily constitute or imply its endorsement, recommendation, or favoring by the United States Government or any agency thereof. The views and opinions of authors expressed herein do not necessarily state or reflect those of the United States Government or any agency thereof.

\author{
PACIFIC NORTHWEST LABORATORY \\ operated by \\ BATTELLE \\ for the \\ UNITED STATES DEPARTMENT OF ENERGY \\ under Contract DE-AC06-76RLO 1830
}

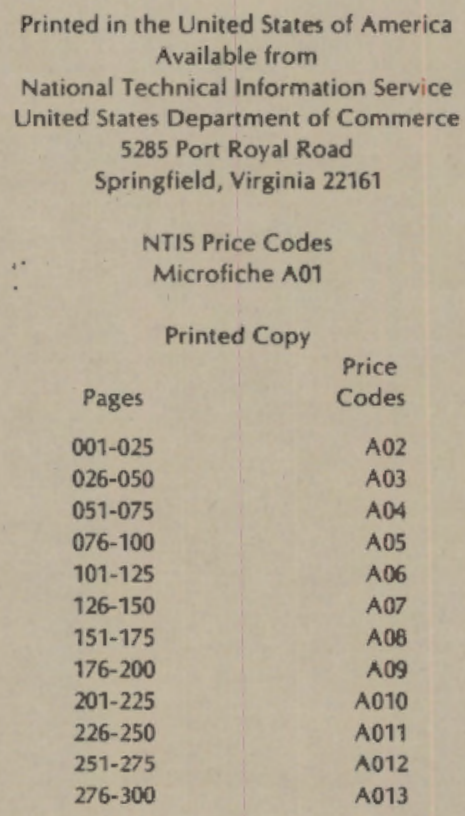


PNL -5465

UC-70

RADAR LOCATION EQUIPMENT

DEVELOPMENT PROGRAM

PHASE I

G. A. Sandness

K. C. Davis

June 1985

Prepared for

the Westinghouse Electric Corporation

under a Related Services Agreement

with the U.S. Department of Energy

Contract DE-AC06-76RLO 1830

Pacific Northwest Laboratory

Richland, Washington 99352 

SUMMARY

The work described in this report represents the first phase of a planned three-phase project designed to develop a radar system for monitoring waste canisters stored in a thick layer of bedded salt at the Waste Isolation Pilot Plant (W]PP) near Carlsbad, New Mexico. The canisters will be contained in holes drilled into the floor of the underground waste storage facility. It is expected that heat and pressure gradients in the surrounding salt will. over a period of years, slightly displace the canisters from their original locations. A monitoring procedure needed to determine the location and radar attitude (tilt angle) of each buried canister. It is hoped that these Measurements can be made to accuracies of $\pm 5 \mathrm{~cm}$ and $\pm 2^{\circ}$, respectively.

The initial phase of this project was primarily a feasibility study. Its principal objective was to evaluate the potential effectiveness of the radar method in the planned canister monitoring application. Its scope included an investigation of the characteristics of radar signals backscattered from waste canisters, a test of preliminary data analysis methods, an assessment of the $e^{\varepsilon f e c t s}$ of salt and bentonite (a proposed backfill material) on the propagation of the radar signals, and a review of current ground-penetrating radar technology.

A laboratory experiment was performed in which radar signals were backscattered from simulated waste canisters. The radar data were recorded by a digital data acquisition system and were subsequently analyzed by three different computer-based methods to extract estimates of canister location and $t i 1 t$. Each of these methods yielded results that were accurate within a few centimeters in canister location and within $1^{\circ}$ in canister tilt. Measurements were also made to determine the signal propagation velocities in salt and bentonite (actualiy a bentonite/sand mixture) and to estimate the signal attenuation rate in the bentonite. Finally. a product survey and a literature search were made to identify available ground-penetrating radar systems and alternative antenna designs that may be particularly suitable for this unique application.

follows:

The principal conclusions to be drawn from this initial work are as

1) The radar sensing method, together with a digital data analysis technique, is capable of measuring canister location and tilt to better than the desired level of accuracy in air and, presumably, in any other uniform dielectric medium.

2) The in situ tests planned to be conducted under Phase 2 are needed to confirm the capability of the radar technique to provide the necessary monitoring capability in the actual containment media.

3) The use of bentonite as a backfill material may cause difficulties, but these difficulties can probably be overcome by utilizing oblique radar viewing angles. The formation of brine in the salt surrounding a canister could constitute the most serious impediment to the use of the radar method, but it is not known whether significant brine-forming moisture concentrations will actually occur. 
4) An operational radar monitoring system can be largely assembled from available radar and data acquisition components; however, it may be necessary to develop a specialized radar antenna by modifying an existing basic design. 


\section{CONTENTS}

SUMMARY

1.0 INTRODUCTION

$\begin{array}{ll}1.1 & \text { BACKGROUND } \\ 1.2 & \text { PROJECT OBJECTIVES }\end{array}$

1.3 PHASE 1 OBJECTIVES

2.0 PROCEDURES AND RESULTS . . . . . . . . . . . . . . 5

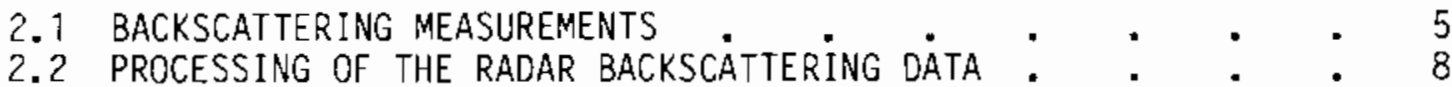

2.2.1 Direct Visual Analysis. . . . . . . 10

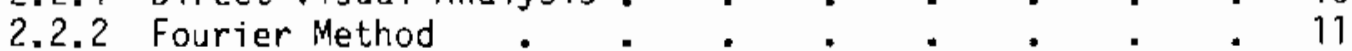

2.2.3 Waveform Correlation Method: . . . . . . 13

2. 3 RADAR SIGNAL PROPAGATION IN SALT AND BENTONITE $\quad \cdot \quad \cdot \quad \cdot 9$

2.3.1 Available Data . . . . . . . . 20

2.3.2 Measurements on Sait and Bentonite : $: \quad . \quad$ : 21

2.3.3 Reflection and Refraction . . . . . . 22

3.0 GPR HARDWARE . . . . . . . . . . . . . . . 27

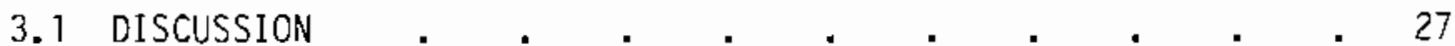

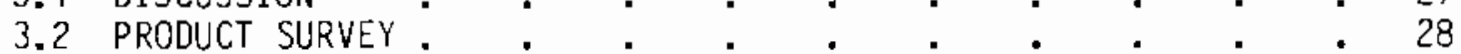

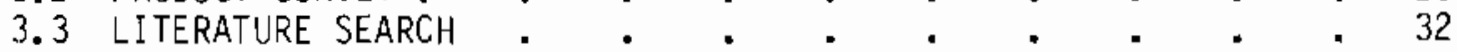

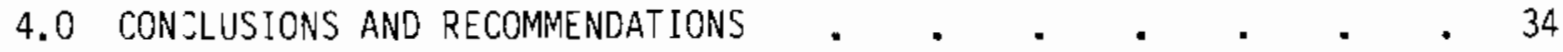

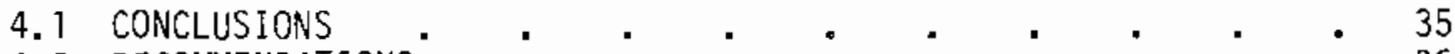

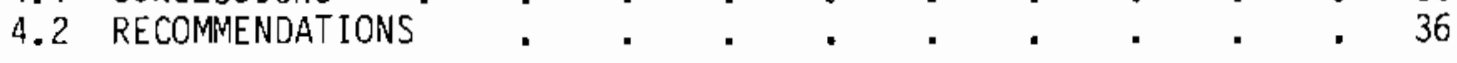

REFERENCES . . . . . . . . . . . . . . . . . . . 39

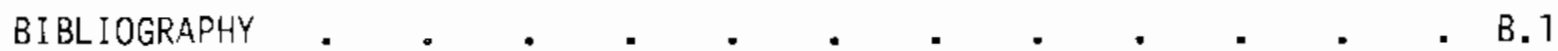

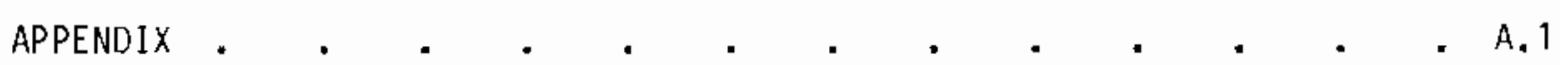




\section{FIGURES}

1.1 The expected designs of the WIPP waste canister and overpack canister

2.1 A plan view of the experimental arrangement for backscattering measurements

2.2 Photographs showing the experimental setup for radar backscattering measurements on a simulated waste canister.

2.3 Radar profiles showing backscattered signals from the simulated waste canister at a $0^{\circ}$ tilt angle.

2.4 Radar profiles showing backscattered data from the simulated waste canister at tilt angles of $0^{\circ}$ (top), $5^{\circ}$ (middle), and $10^{\circ}$ (bottom)

2.5 Illustration of the direct measurement of canister tilt angle using the slope of a flat section of the reflection pattern

2.6 Intensity-modulated images showing phase slope values

for signals backscattered from the simulated waste canister at tilt angels of $0^{\circ}$ (left) and $10^{\circ}$ (right)

2.7 Calculated asymetry coefficient as a function of canister tilt angle for both test canisters . . . . . 14

2.8 The geometry for correlation analysis . . . . . . 14

2.9 Correlation data for the simulated waste canister at tilt angles of $0^{\circ}$ (left) and $10^{\circ}$ (right).

2.10 Correlation data for the simulated overpack canister at a tilt angle of $8.5^{\circ}$

2. 11 The geometry used to calculate the canister tilt angle using the correlation data displayed in Figures 2.9 and 2.10

2.12 Examples of ray paths for radar signals backscattered from a canister buried in salt with bentonite/sand backfill material. A possible thin clay layer is also illustrated

2.13 Refraction of a radar signal specularly reflected from the canister pintle.

3.1 GPR systems produced by GSSI (left) and OYO 
3.2 Gulf applied research system for highway inspection . . . 29

3.3 The Penetradar GPR system . . . . . . . . . . 30

A. 1 The measured complex dielectric constant of a clay soil with a moisture content of $10 \%$ by weight . . . . A.2

A.2 Empirical relationship between $\varepsilon_{r}^{\prime}$ and the volumetric moisture content of mineral soils = . . . . . . A.3

A.3 Attenuation rate versus moisture content for two soils and two signal frequencies. . . . . . . A.4

A.4 Attenuation rate versus frequency for two soils and two levels of volumetric moisture content . . . . A.5

\section{TABLES}

2.1 Parameters relating to radar signal propagation in selected materials

3.1 Summary of the characteristics of commercial GPR systems

3.2 Summary of antenna characteristics . . . . . . . . . . . 33

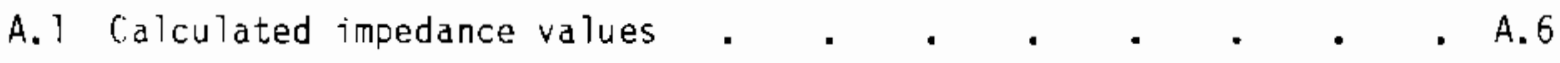


$\cdot$

, 


\subsection{INTRODUCTION}

\subsection{BACKGROUND}

This study was conducted in support of the Waste Isolation Pilot Plant (WIPP) project now underway at a site near Carlsbad, New Mexico. Under this project, high- and low-level nuclear waste materials will be stored underground in a facility located in a thick layer of bedded salt. The work described in this report relates specifically to the storage of the high-level radioactive waste materials. These materials will be encapsulated in specially designed metal canisters which will be emplaced in cylindrical holes drilled in the floor of the mined salt facility.

The locations and orientations of the waste storage canisters will be precisely determined and recorded during the emplacement process in order to ensure that the canisters can be adequately monitored and that they can be removed from their holes if necessary. It is expected, however, that the combined effects of high pressure gradients and high temperatures in the waste storage areas will result in significant rates of flow or creep in the volume of salt immediately surrounding the storage facility.

Over a period of years, this phenomenon will produce a substantial amount of room closure and will make it difficult to maintain the reference points needed to precisely locate the buried waste canisters. Furthermore, it may be impossible to accurately predict the displacement (translation and rotation) that a given canister may undergo in this environment. An inability to routinely obtain this information would be unacceptable for at least two reasons: 1) it would involve the risk of damaging canisters during possible reaming-out operations in the storage rooms and 2) it would require an increased amount of radiation shielding for operator safety during canister retrieval operations. A sensing device capable of measuring the position and orientation (tilt) of each buried waste canister is needed to eliminate or at least minimize these potential problems.

\subsection{PROJECT OBJECTIVES}

As shown in Figure 1.1, the canisters are expected to be of two types: 1) a titanium-walled canister containing nuclear fission products, and 2) a larger carbon steel overpack canister containing a stainless steel inner canister. The sensing system to be developed should be capable of locating these canisters in vertical boreholes spaced $2.1 \mathrm{~m}$ apart in a drift that is approximately $5.5 \mathrm{~m}$ wide $\times 5.5 \mathrm{~m}$ high. In terms of measurement accuracy, the sensing system should be capable of locating the top of a canister lifting pintle to within $\pm 5 \mathrm{~cm}$ and of determining the canister tilt and azimuth angles to within $\pm 2^{\circ}$.

The operational characteristics of the system should be such that it will not require excessive setup time, continuous hands-on attention by an operator, or physical penetration of the salt. Finally, it should include a data acquisition system that will operate largely in an automated mode and produce digital data sets suitable for immediate computer processing and analysis. 

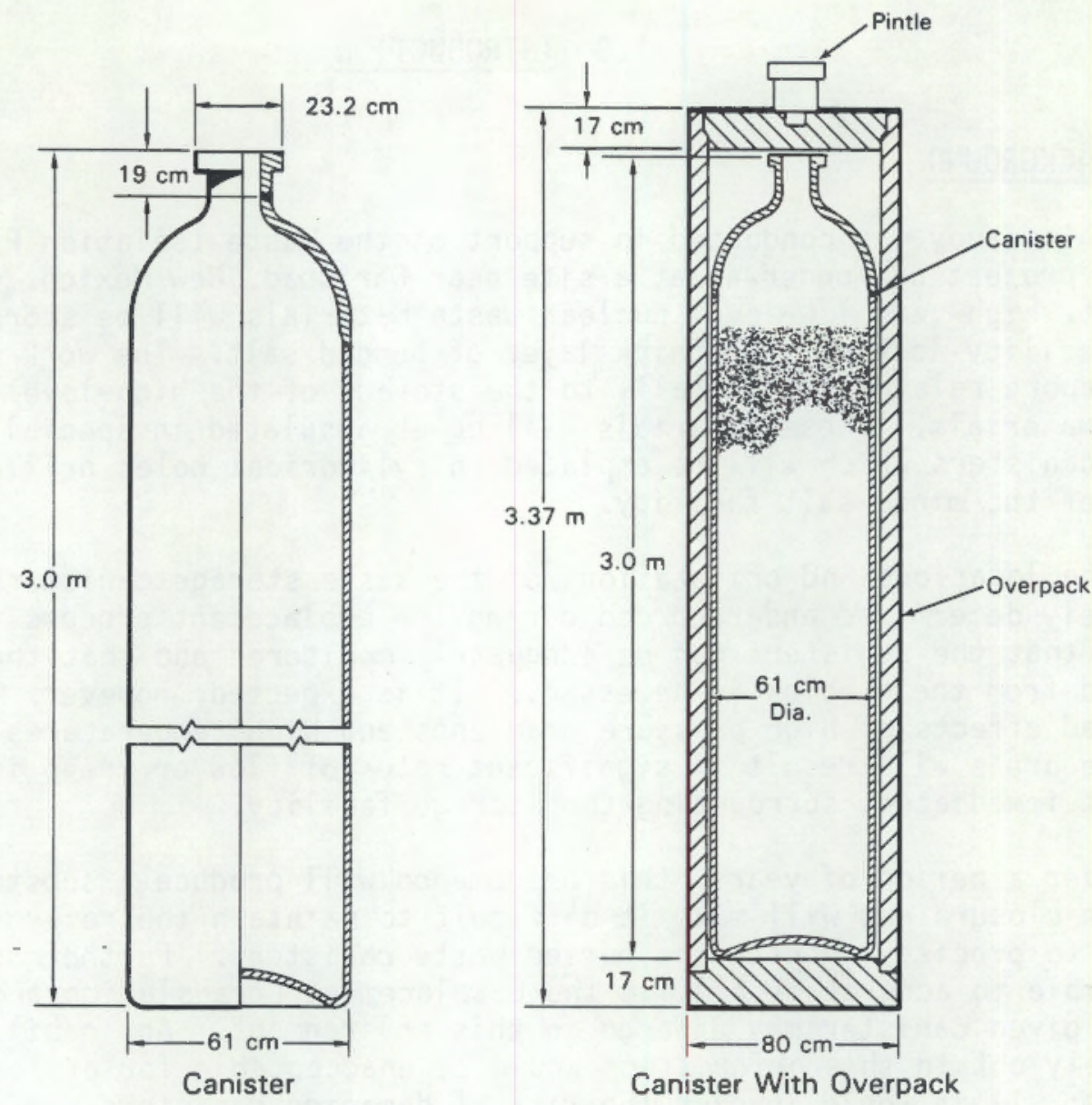

FIGURE 1.1. The expected designs of the WIPP waste canister and overpack canister.

This project is focused specifically on a radar technique to accomplish these goals. The use of radar to detect and characterize targets contained in a material medium is a relatively new, but effective, sensing method. Systems have been developed for a wide variety of ground-penetrating applications such as mapping underground pipes and utility lines, detecting and mapping shallow deposits of buried chemical and nuclear waste materials, and measuring ice thickness. In terms of their basic operating principles, these systems are similar to the more familiar radar systems used for tasks such as military target detection, marine and aircraft navigation, and aircraft traffic control. The most distinctive design characteristic of the ground-penetrating radar (GPR) systems is their ability to detect targets at extremely short ranges--typically a few centimeters to a few tens of meters.

A GPR system appears to offer several attributes that are significant in this particular application:

- It offers relatively high spatial resolution in comparison to other potentially applicable sensing techniques such as electromagnetic induction (metal detectors), magnetic, and acoustic methods. 
- It is expected to be able to perform its function without the aid of any auxiliary components (signal sources, reflectors, etc.) that would have to be attached to the canisters.

- Its compact physical configuration is well suited to fast, efficient operation in a relatively confined area.

- Its output can be displayed in an easily understood pictorial form. It can also be computer processed to maximize the amount of extractable target information.

- Its performance in characterizing an individual canister will not be degraded or complicated by the proximity of other canisters.

A three-phase R\&D project has been defined to pursue the goal of developing an optimum GPR system for monitoring the locations and orientations of the high-level waste canisters. The phases are briefly described as follows:

Phase 1 - Perform a feasibility and scoping study to assess the probable effectiveness of the radar method.

Phase 2 - Perform on-site tests using a preliminary radar system configuration to assess actual radar performance in the WIPP environment.

Phase 3 - Design, construct, and demonstrate a complete, operational, radar system for canister monitoring at the WIPP site.

The phasing of this project plan provides two break points at which it will be possible to evaluate the results of the preceding phase(s) in terms of the probability of success in meeting the final project goals. This report represents the completion of work under Phase 1.

\subsection{FHASE 1 OBJECTIVES}

This initial work had two main objectives. The first was to make a preliminary assessment of the feasibility of using the radar method to measure the location and orientation of waste canisters in salt. Addressing this objective involved the study of three major topics:

1) the characteristics of radar signals backscattered from waste canisters.

2) methods of processing the backscattered radar signals to extract canister position information, and

3) the effects of salt and a bentonite/sand mixture (a proposed backfi\}l material) on the propagation of radar signals.

The second objective was to develop a research plan (proposal) for the in situ tests to be performed under Phase 2. This aspect of the study involved a brief investigation of alternative radar hardware designs. 
The next section of this report describes our Phase 1 research activities and the results that we obtained. The last section contains our conclusions and recommendations relating to Phase 2 and the overall project plan. A detailed proposal for follow-on work under Phase 2 will be presented as a separate document. 


\subsection{PROCEDURES AND RESULTS}

\subsection{BACKSCATTERING MEASUREMENTS}

The use of backscattered radar signals to determine the location and orientation of a buried waste canister or overpack canister will require that two conditions be satisfied:

1) the backscattered signals must be sufficiently strong to allow the signals to be distinguished from noise and clutter, ano

2) the characteristics of the backscattered signal must be strongly dependent on the position and orientation of the canister relative to the radar antenna.

The degree to which these conditions will be satisfied in the WIPP environment will be determined by several factors. Specifically, the amplitude of the backscattered signals will depend on the power and sensitivity of the radar unit, the backscattering properties of the canisters, and the composition and distribution of the materials in and around the emplacement holes. The signal characteristics will be determined by the shape of the transmitted waveform, the shapes and sizes of the canisters, and the backscattering geometry.

The scope of this Phase 1 effort did not permit a study of the effects of these factors under operational conditions (this will be done in Phase 2), but it did include a laboratory experiment which provided much useful data. This experiment involved the use of an available raciar system to measure highfrequency radar signals backscattered from simulated canisters.

Each of the test canisters was fabricated from a larse, steel, liquid gas container. The upper end of each of these containers had a rounded shoulcier that closely liatched that of the WIPP waste canister design that was current when the test canisters were being constructed. That design has since been superceded by one which specifies a hemispherical shoulder shape as illustrated in Ficure 1.1. In order to match the shape of an overpack canister, one of the containers was modified by adding a flat, square-shouldered, end cap to its ufper end. A WIPP-standard pintle assembiy was welded to the top of each of the containers. The resulting targets had the following dimensions:

$\begin{array}{lcc} & \text { Diameter }(\mathrm{cm}) & \text { Total Length (ii) } \\ \text { Simulated Waste Canister } & 50.5 & 1.55 \\ \text { Simulated Overpack Canister } & 55.0 & 1.52\end{array}$

The differences in the dimensions of the simulated and actual canisters were not important in the test because our purpose was to assess the basic information content of the radar signal rather than to determine its detailed shape. More important is the fact that this experiment was performed in air rather than in salt. In the latter medium, the wavelength of each spectral component of the radar signal would be shortened by a factor of approximately 2.5 (the square root of the dielectric constant of salt). Thus, the ratio of the canister diameter to the average signal wavelength was smaller in this 
test case than it would be in the in situ application. Because the spatial information (target location and orientation) contained in a radar signal is less at long wavelengths than at short wavelengths, this experiment represented a worst-case test of the radar monitoring method.

The radar unit used for this test was a Geophysical Survey Systems, Inc. (GSSI) System 7 with a Model 101C. 900-MHz antenna. This unit is an impulse-type radar operating on the principle of time domain reflectometry (range proportional to signal travel time). The backscattered radar signals (waveforms) were digitized by a microcomputer-based data acquisition unit and recorded in digital form on a cartridge magnetic tape.

The backscattering measurements were made using a geometrical arrangement such that the axis of the target canister was positioned horizontally at a height of $1.43 \mathrm{~m}$ above the laboratory floor. The canister was supported by a radar-transparent box fabricated from sheets of rigid polystyrene foam.

An apparatus was also constructed to move the GPR antenna along a set of straight, horizontal lines located in a vertical measurement plane. This arrangement permitted the collection of radar returns from the simulated canisters over a substantial range of incident and azimuth angles. A vertical column mounted on a wheeled carriage allowed the GPR antenna to be positioned on the colurin at 10 regularly spaced, $10-\mathrm{cm}$ intervals starting at a height of $1.0 \mathrm{~m}$ above the floor. The carriage was moved along a horizontal rail which fixed the location of the measurement plane. With the antenna centered on the canister axis, the distance from the antenna to the canister pintle was $124 \mathrm{~cm}$. The range of horizontal motion was $3 \mathrm{~m}$, providing a $0.9-m-h i g h$ by 3-m-long measurement plane. The carriage and verical column were constructed of wood and plastic to minimize radar reflections from the apparatus. The general setup and details of the components are shown in Figures 2.1 and 2.2.

During each traverse of the measurement plane, the antenna carriage was pulled manually along the horizontal guide rail. The horizontal position of the radar antenna was determined by assuming a constant carriage velocity; thus, any irregularities in that motion produced errors in the positions at which the data were assumed to have been taken. Analysis of the data indicated that a few scans exhibited position errors of as much as $15 \mathrm{~cm}$, but that the typical horizontal positioning accuracy was approximately $5 \mathrm{~cm}$. Vertical positioning of the antenna, determined by fixed locating holes in the vertical column, was accurate to less than $0.5 \mathrm{~cm}$.

Backscattered radar waveforms were collected at a rate of approximately 4 per second as the radar antenna was pulled along the $3-m-l o n g$ track. The rate of motion was adjusted so that approximately 100 waveforms were collected on each traverse. The analog-to-digital conversion rate was set to produce 200 samples within an effective time interval of $35 \mathrm{nsec}$. The range represented by the last digitized value in each waveform was $4.0 \mathrm{~m}$.

The radar measurements were made in a large open area so that return signals from other objects did not interfere with the identification of characteristic return signals from the pintle and front surfaces of the canisters. Radar signals reflected from the floor did interfere with 


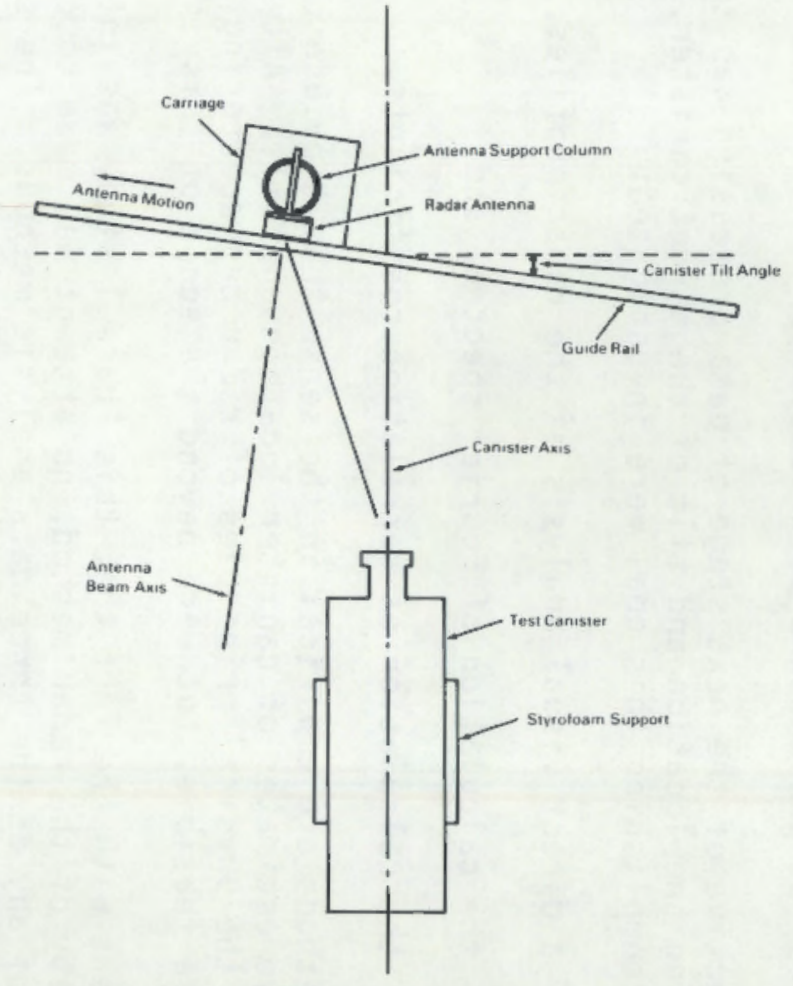

FIGURE 2.1. A plan view of the experimental arrangement for backscattering measurements
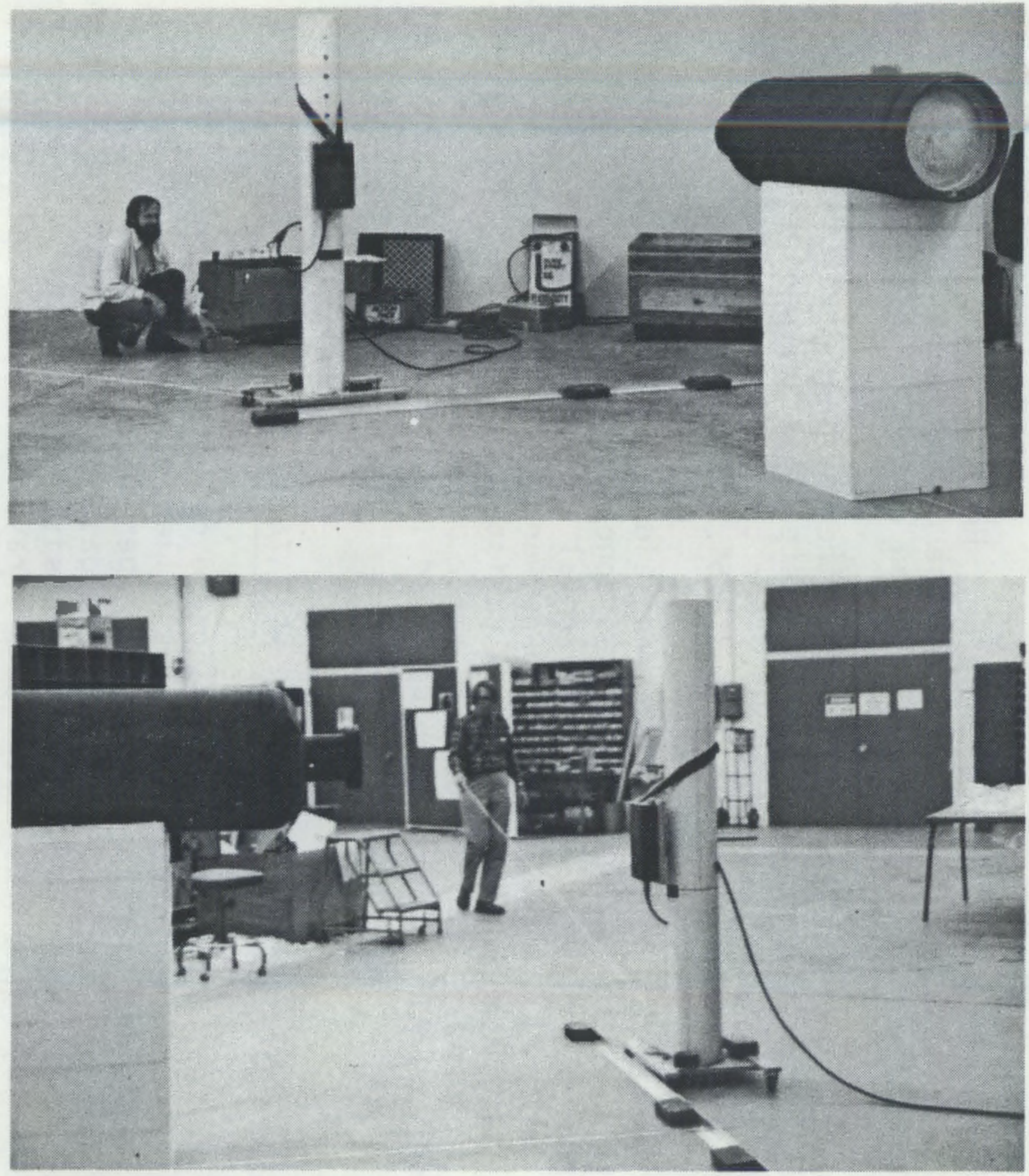

FIGURE 2.2. Photographs showing the experimental setup for radar backscattering measurements on a simulated waste canister 
reflections from the far end of the canister. However, identification of the far end of the canister was not a goal of these measurements since the test geometry could not fully approximate that of an actual emplaced waste canister.

Canister tilt was simulated by rotating the carriage track relative to the target axis. For the round-shouldered waste canister, measurements were made at "tilt" angles of $0^{\circ}, 5^{\circ}$, and $10^{\circ}$. For the square-shouldered overpack canister, measurements were made at $0^{\circ}$ and $8.5^{\circ}$.

At each tilt angle, two scans were made along the 3-m track at each of the ten possible antenna heights. This was done first with the antenna polarization (electric field vector) horizontal and again with the antenna polarization vertical. A total of 120 scans were made using the waste canister and 80 scans were made using the overpack canister. A series of scans were also made on a spherical target to check radar performance and the behavior of the test setup. All the data were recorded in digital form and converted to a standard format for computer analysis.

\subsection{PROCESSING OF THE RADAR BACKSCATTERING DATA}

The first step in computer processing the collected data was a linear, along-track interpolation of the waveforms collected along each 3-m traverse. This procedure yielded exactly 100 waveforms per traverse. Figure 2.3 is a photographic display of one of the resulting data sets. Each of the 20 images, or radar profiles, in this figure is an intensity-modulated display of a $200-$ row (sample) by 100 column (waveform) data array. The horizontal dimension in each profile corresponds to distance along the horizontal traverse line $(0-3 \mathrm{~m})$. The vertical dimension corresponds to signal travel time $(0-27 \mathrm{nsec})$ or range $(0-4 \mathrm{~m})$. The 20 profiles represent two traverses at each of the 10 antenna heights (at one antenna polarization).

The objective of the next stage of data processing was to extract numerical values defining the location and tilt of the target canister. Three different methods of accomplishing this goal were investigated:

1) a direct visual analysis of the radar profiles,

2) the calculation of Fourier spectra, and

3) the calculation of correlation coefficients.

These methods are empirical in the sense that they use only the measured data to derive estimates of canister location and tilt. Alternative approaches which invoke the physical principles of radar backscattering to compute target signatures are feasible, but were beyond the scope of this initial study.

Consistent with the fact that this Phase 1 study was intended to assess the feasibility of the radar method, no attempt was made to complete the development of any of the three data analysis methods. The selection of a data processing method for operational use should not be made until additional tests can be performed and until further investigation of alternative methods can be completed. These actions are expected to constitute a major part of Phase 2 and perhaps even a part of Phase 3. 


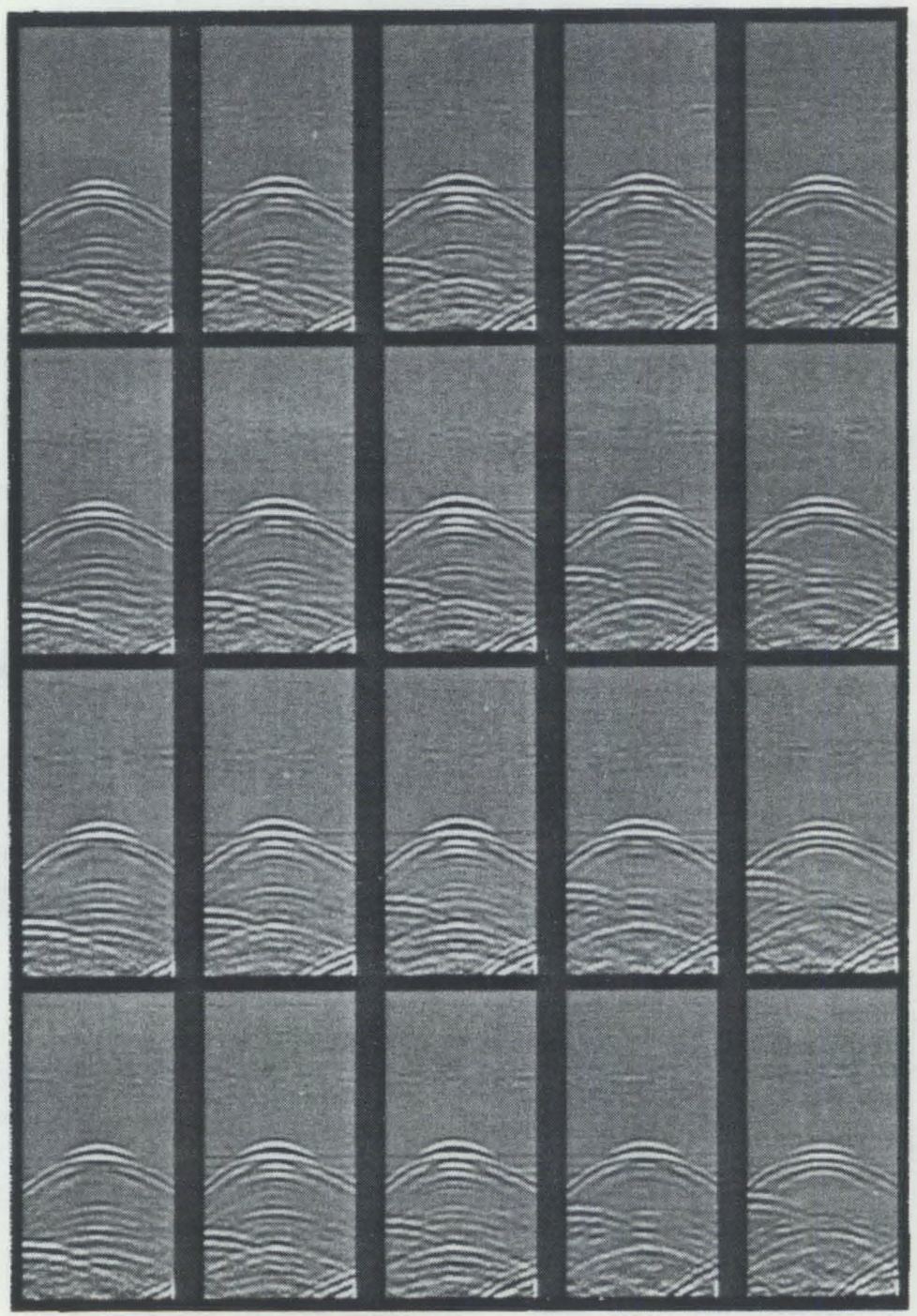

FIGURE 2.3. Radar profiles showing backscattered signals from the simulated waste canister at a $0^{\circ}$ tilt angle. The 20 profiles represent two traverses at each of 10 antenna heights. Oata collected at a height of $1.0 \mathrm{~m}$ is in the upper left corner; data from $1.9 \mathrm{~m}$ is in the lower right corner. 


\subsubsection{Direct Visual Analysis}

An examination of sets of profiles such as those shown in Figure 2.3 indicates that it is relatively easy to determine the height of the test canister's axis. As expected in this test, the profiles collected at a height of $1.4 \mathrm{~m}$ most closely represented a convergence of the antenna path with the target axis. Segments of six such profiles are shown in Figure 2.4. The data contained in these profiles span the travel time interval between 6.9 and $14.0 \mathrm{nsec}$ and represent backscattering from the simulated waste canister at both vertical and horizontal antenna polarizations (left and right sides of the figure, respectively). From top to bottom, these profiles correspond to canister tilt angles of $0^{\circ}, 5^{\circ}$, and $10^{\circ}$.
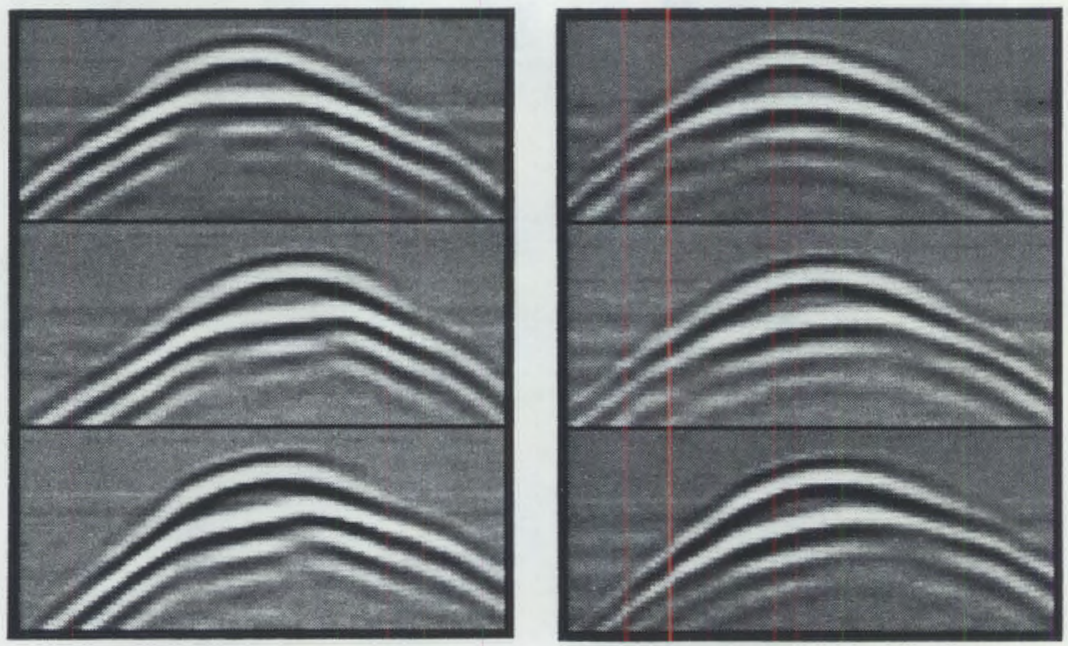

FIGURE 2.4. Radar profiles showing backscattered data from the simulated waste canister at tilt angles of $0^{\circ}$ (top), $5^{\circ}$ (middle), and $10^{\circ}$ (bottom). The left and right sets of profiles show vertically polarized and horizontally polarized data, respectively.

In each of the profiles, an indication of the tilt angle is given by the symmetry (or asymmetry) of the reflection pattern and by a distinctive flattening of the reflection pattern in the central part of the image. As illustrated in Figure 2.5, the latter feature can provide quantitative measurements of the location and tilt of the canister axis. The desired values are obtained by measuring the midpoint and slope of the flattened section. A vertical exaggeration in the image scale must be taken into account in calculating the slope. In the example shown, the apparent location of the canister axis is offset from the center of the profile because of a variation in the speed at which the antenna was pulled along the traverse line. A tabulation of the canister tilt angles measured by this method is as follows:

Actual Tilt Angles

$\begin{array}{lllll} & -\frac{0^{\circ}}{0^{\circ}} & -\frac{5.0^{\circ}}{5.2^{\circ}} & -\frac{8.5^{\circ}}{-} & -\frac{10.0^{\circ}}{9.8^{\circ}} \\ \text { Waste Canister } & 0^{\circ} & -- & 8.4^{\circ} & -\cdots\end{array}$




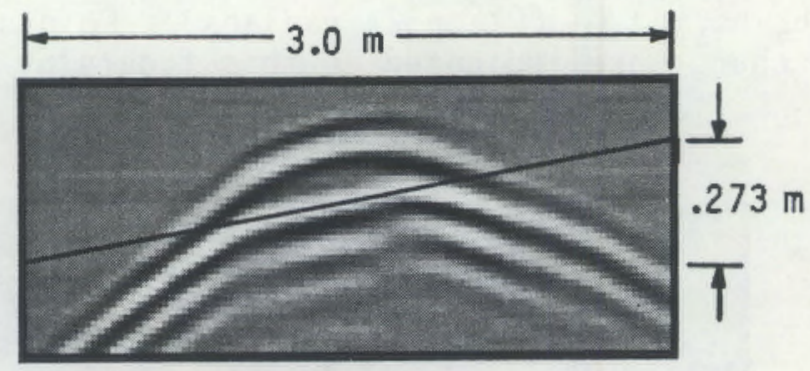

FIGURE 2.5. Illustration of the direct measurement of canister tilt angle using the slope of a flat section of the reflection pattern. The tilt angle is $\theta=\tan ^{-1}(.273 / 3)=.5.2^{\circ}$.

The uncertainty in each of these values is $\pm 0.5^{\circ}$; thus this method appears to be sufficiently accurate. It is not clear, however, that the reflection patterns obtained from buried canisters will exhibit the same diagnostic features that are present in the patterns obtained in air.

\subsubsection{Fourier Method}

All of the target-related information available in the backscattered radar signals is contained in the shapes of the waveforms. No additional information can be generated by transforming the waveforms from the time domain into the frequency domain. On the other hand, a frequency-domain presentation of the waveforms offers new possibilities for data interpretation.

The Fourier transform of a time function $f(t)$ (e.g., an individual radar waveform) is given by

$$
F(v)=\int_{-\infty}^{\infty} f(t) e^{-j 2 \pi v t} d t
$$

where $v$ is the frequency and $F(v)$ is the complex frequency spectrum. The application of this transform to our digital radar data was accomplished by means of a Fast Fourier Transform (FFT) computer algorithm. Each 200-point time-domain waveform was supplemented by 56 trailing zeros to make the number of points equal to a power of two, then transformed to a 128-point frequency spectrum. Each complex spectral component could be characterized by the product of a magnitude term and a phase term. For a given radar traverse, or profile, either the magnitude values or the phase values constituted a new two-dimensional array which could be displayed in the same intensity-modulated form as the radar profiles shown in Figures 2.3-2.5. An example is Figure 2.6 which shows the phase values for the radar signals backscattered from the simulated waste canister at 10 antenna heights and tilt angles of $0^{\circ}$ and $10^{\circ}$. Here the horizontal dimension again corresponds to distance along the 3-m traverse 1 ine, but the vertical dimension corresponds to frequency $(0-2824$ $\mathrm{MHz}$ ) rather than to range or travel time. Similar images were obtained by displaying the corresponding magnitude values. 
The values displayed in Figure 2.6 are actually not the raw phase angles calculated by the FFT, but rather values of the slope of the phase spectrum produced by allowing the phase spectrum to be continuous (i.e., to take values greater than the normal $0-360^{\circ}$ or $2 \pi$ radians). Points of maximum and minimum rates of phase change are indicated in this figure by white and black tones, respectively.
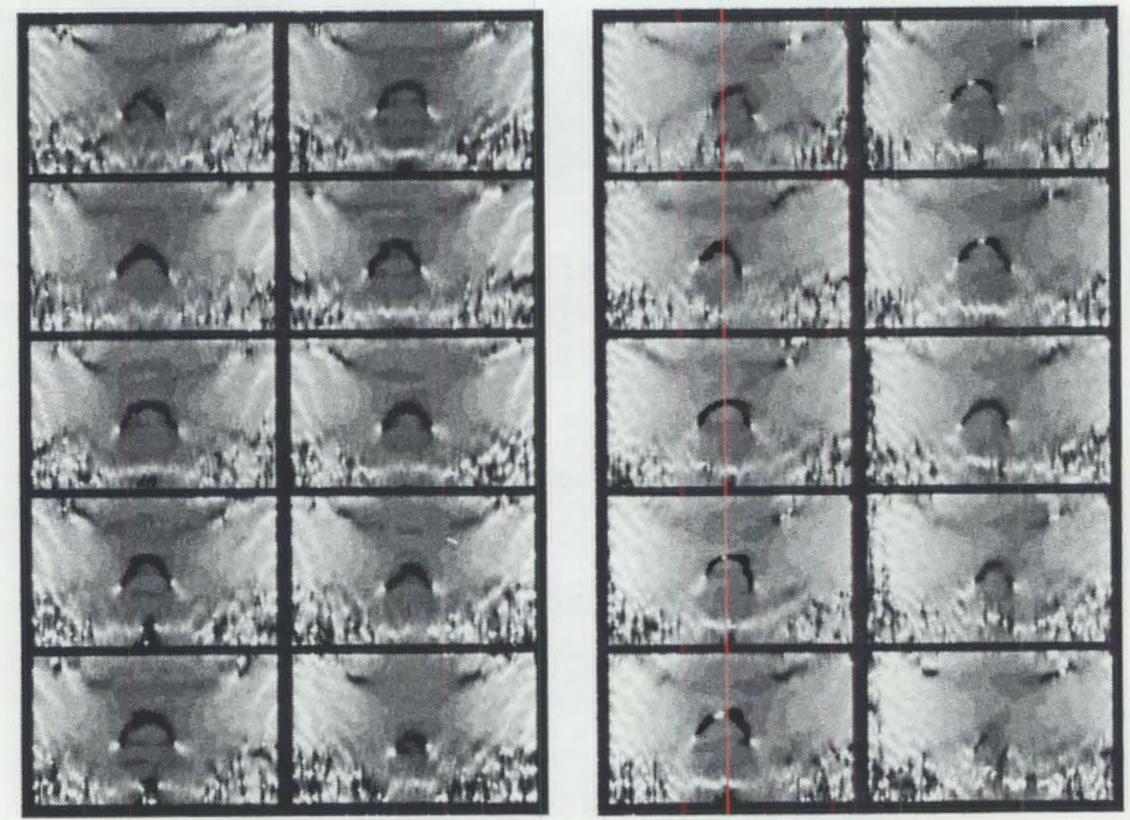

\section{FIGURE 2.6. Intensity-modulated images showing phase slope values for signals backscattered from the simulated waste canister at tilt angles of $0^{\circ}$ (left) and $10^{\circ}$ (right).}

Several features which may potentially convey information about the location and orientation of the target canister are present in these images. An example is the small, arcuate, black pattern located near the center of each image. This pattern, representing frequencies at which the rate of change of phase angle is a minimum, is apparently associated with reflections from the near (pintle) end of the canister. The shapes of the patterns produced by the round-shouldered waste canister and the square-shouldered overpack canister, respectively, suggest that the patterns are associated with specular reflections from appropriately oriented segments of the canister surfaces. Bright spots located at the ends and, in some cases, near the center of the black patterns, suggest unique frequency responses at the outer edges of the canister shoulder and on the canister axis.

A second feature, which may be more directly usable for deriving canister tilt angles, is a marked lateral asymmetry in the phase patterns of Figure 2.6. The phase pattern in a given image tends to be the same on either side of the image centerline only in those cases where the canister tilt angle is $0^{\circ}$. The asymmetry is most pronounced at antenna locations greater than $\pm 0.5 \mathrm{~m}$ from the centerline. This suggests that the features of interest are associated 
with non-specular components of the backscattered signals and that the phases of these components depend on the tilt of the canister relative to the radar antenna. Thus, a quantitative measure of the asymmetry of a measured phase pattern may provide a good estimate of the canister tilt angle. To test this concept, an "asymmetry coefficient" was defined as described in the next paragraph.

Assume that a measured radar profile contains $M$ waveforms and that each waveform contains $\mathrm{N}$ digitized data. After Fourier transformation of each waveform as described above, this data array will be converted to a new array containing $M$ columns (spectra) and $K$ rows (frequency components in each spectrum). Each term in the array will contain the slope of the phase function at the corresponding spectral frequency. The asymmetry coefficient is then

$$
c=\frac{10}{M} \sum_{j=1}^{M / 2}\left(\sum_{i=I_{1}}^{I_{2}} P_{i j} / \sum_{i=I_{1}}^{I_{2}} P_{i, M-j+1}\right)
$$

where $P_{i j}$ is the slope of the phase of the ith frequency component in the $j$ th column. $I_{1}$ and $I_{2}$ define the range of frequencies to be used in calculating the asymmetry coefficient.

Using our measured backscattering data to calculate the asymmetry coefficient gave the results shown in Figure 2.7. This graph suggests that ratioing the phase slope values from opposite sides of the canister axis as described above may provide a sensitive measure of canister tilt.

\subsubsection{Waveform Correlation Method}

This method is based on the expectation that a radar waveform backscattered from an axially symmetric canister at a specific angle relative to the canister axis will be unique. If this is correct, it should be possible to scan a measurement plane to find a set of points at which the backscattered signal is identical to a prerecorded reference waveform obtained at a known backscattering angle. Given such a set of points, it would be only an exercise in trigonometry to determine the location and tilt of the canister. The accuracy of this process could be enhanced by: 1) using additional reference waveforms to map the loci of several backscattering angles, and 2) using target range data in addition to points on the reference plane to compute the canister location and tilt. In a preliminary test of this method, the previously described radar backscattering data were used together with a digital signal correlation technique to identify the reference waveforms.

The geometry of the backscattering measurement is illustrated in Figure 2.8. A waste canister is shown tilted at an angle $\theta$ relative to a measurement reference plane. Radar waveforms corresponding to backscattering angles of $13.5^{\circ}, 25.6^{\circ}$, and $35.8^{\circ}$ were identified in the data set collected at a $0^{\circ}$ tilt angle. These waveforms were extracted and used as reference waveforms for the correlation computations. 


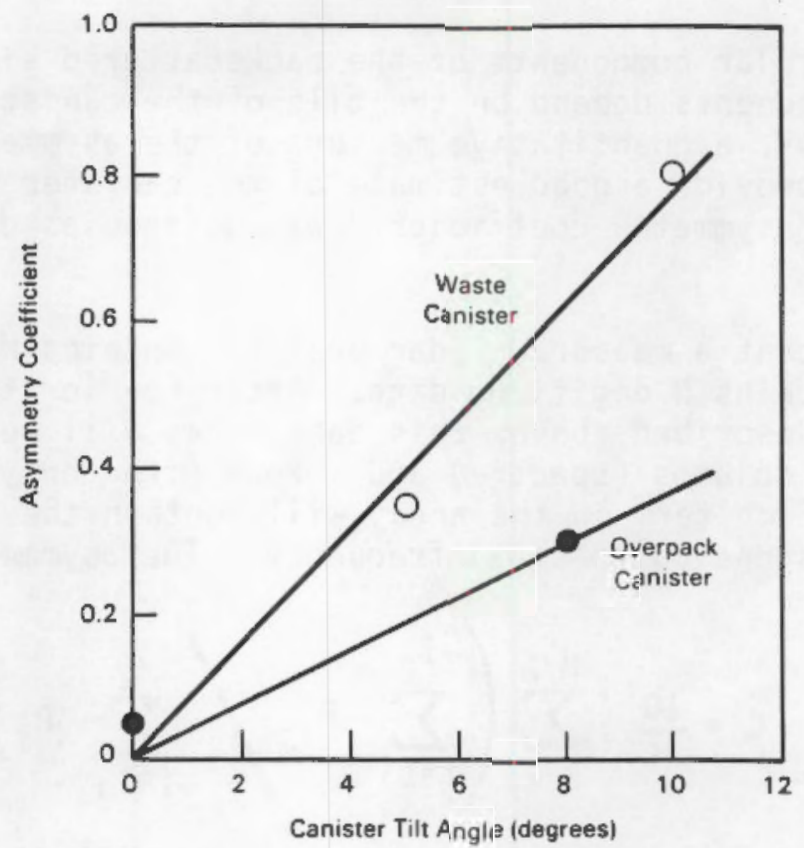

FIGURE 2.7. Calculated asymmetry coefficient as a function of canister tilt angle for both test canisters.

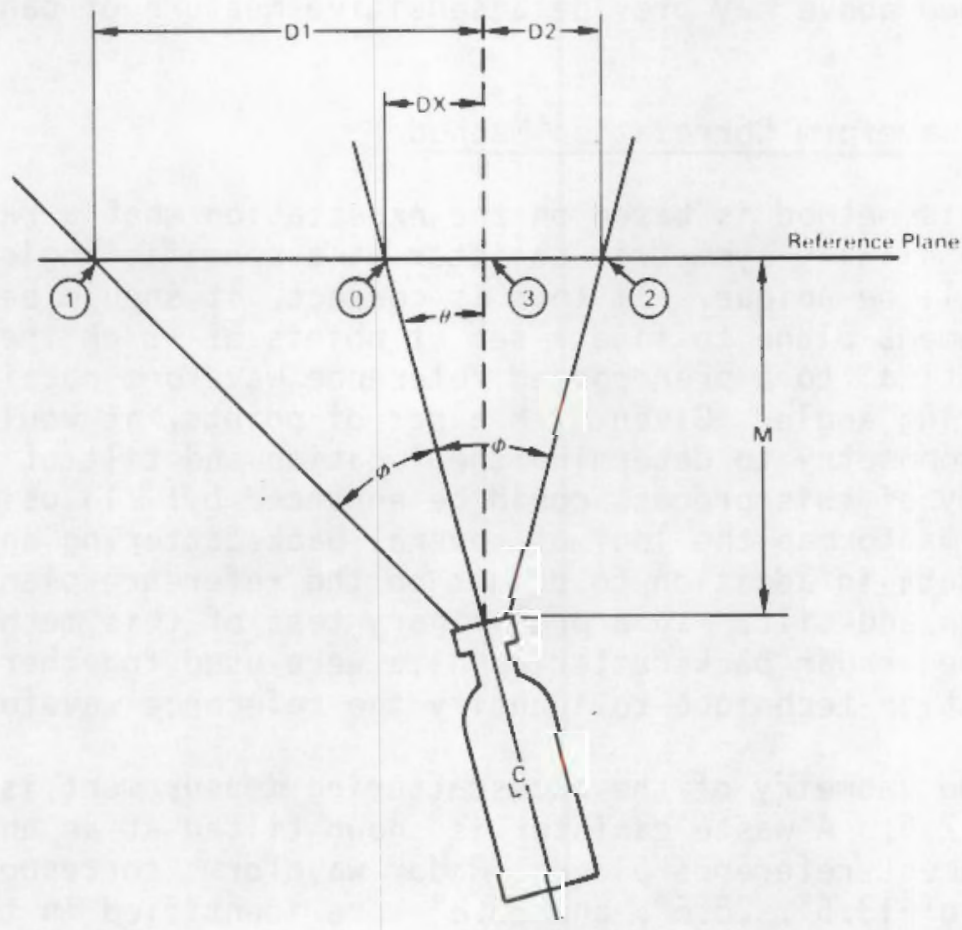

FIGURE 2.8. The geometry for correlation analysis. 
The radar data were recorded as a series of numbers representing the amplitude of the radar return as a function of time. Let the increment of time between samples be $\tau$; then the data obtained at a single antenna location (i.e., a single waveform) may be represented by the series

$$
D_{1}, D_{2}, D_{3}, \ldots D_{j}, \ldots D_{n}
$$

where $D_{j}$ is the signal amplitude at time $j \tau$. A reference waveform extracted from a set of such data collected under known conditions may be represented by the series

$$
R_{1}, R_{2}, R_{3}, \ldots R_{j}, \ldots R_{m}
$$

where $m<n$. The correlation coefficient was calculated in the form

$$
S_{i}=\frac{\sum_{j=1}^{m} R_{j} D_{i+j}}{\sqrt{\sum_{j} R_{j}^{2} \sum_{j}^{2} D_{i+j}^{2}}}
$$

where the index $i$ gives the number of increments by which the reference waveform has been shifted along the data waveform. The RMS terms in the denominator normalize the correlation coefficient.

Figure 2.9 shows calculated correlation coefficients displayed in the usual intensity-modulated format. The horizontal dimension corresponds to distance along the traverse line and the vertical dimension corresponds to range. The horizontal white line in each image shows the $3-\mathrm{m}$ length of the traverse line and represents the range base line. The bright spots, corresponding to high values of the correlation coefficient, show the locations of backscattered waveforms that matched the reference waveforms. Thus, in Figure 2.9, the bright spots indicate the locations of waveforms backscattered from the waste canister at the preselected backscattering angles. Figure 2.10 shows the equivalent results for the overpack canister. The results shown in both of these figures were derived from data collected along the traverse line located $1.4 \mathrm{~m}$ above the floor. Figure 2.11 shows the geometry used to estimate the canister tilt angles from the data displayed in Figures 2.9 and 2.10. Distances from the range base line to the bright spots in the photographs correspond to ranges $R_{1}$ or $R_{2}$ in Figure 2.11. The horizontal separation between spots corresponds to the distance $D_{1}+D_{2}$. Angles measured from the photographs may thus be related directly to the canister tilt angle. The equation relating the measured angle $\Omega$ to the actual canister tilt angle $\theta$ is

$$
\tan \Omega=\frac{\sec (\phi+\theta)-\sec (\phi-\theta)}{\tan (\phi+\theta)+\tan (\phi-\theta)}
$$



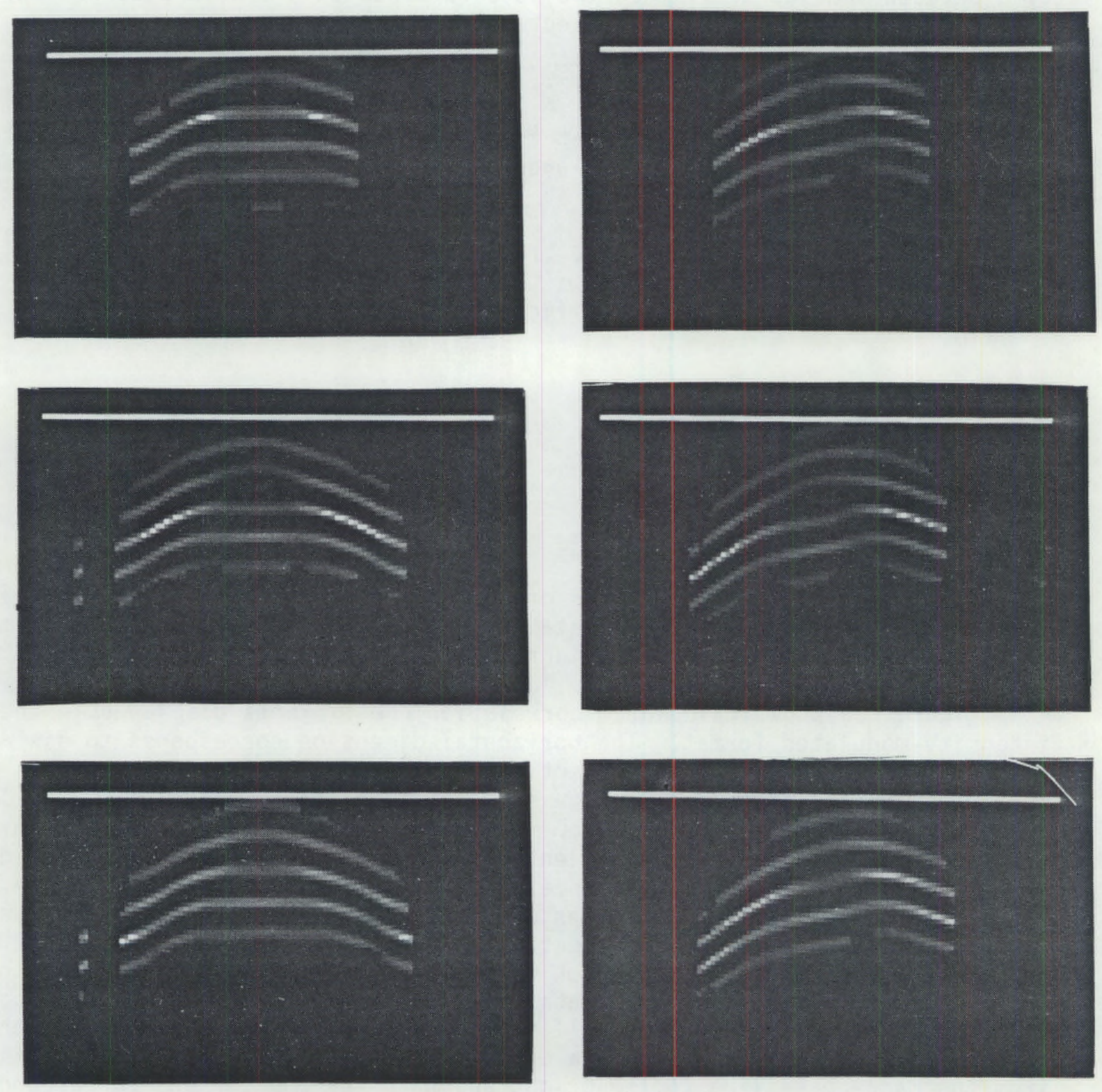

FIGURE 2.9. Correlation data for the simulated waste canister at tilt angles of $0^{\circ}$ (left) and $10^{\circ}$ (right). From top to bottom, the images correspond to reference angles of $13.5^{\circ}, 25.6^{\circ}$, and $35.8^{\circ}$. 

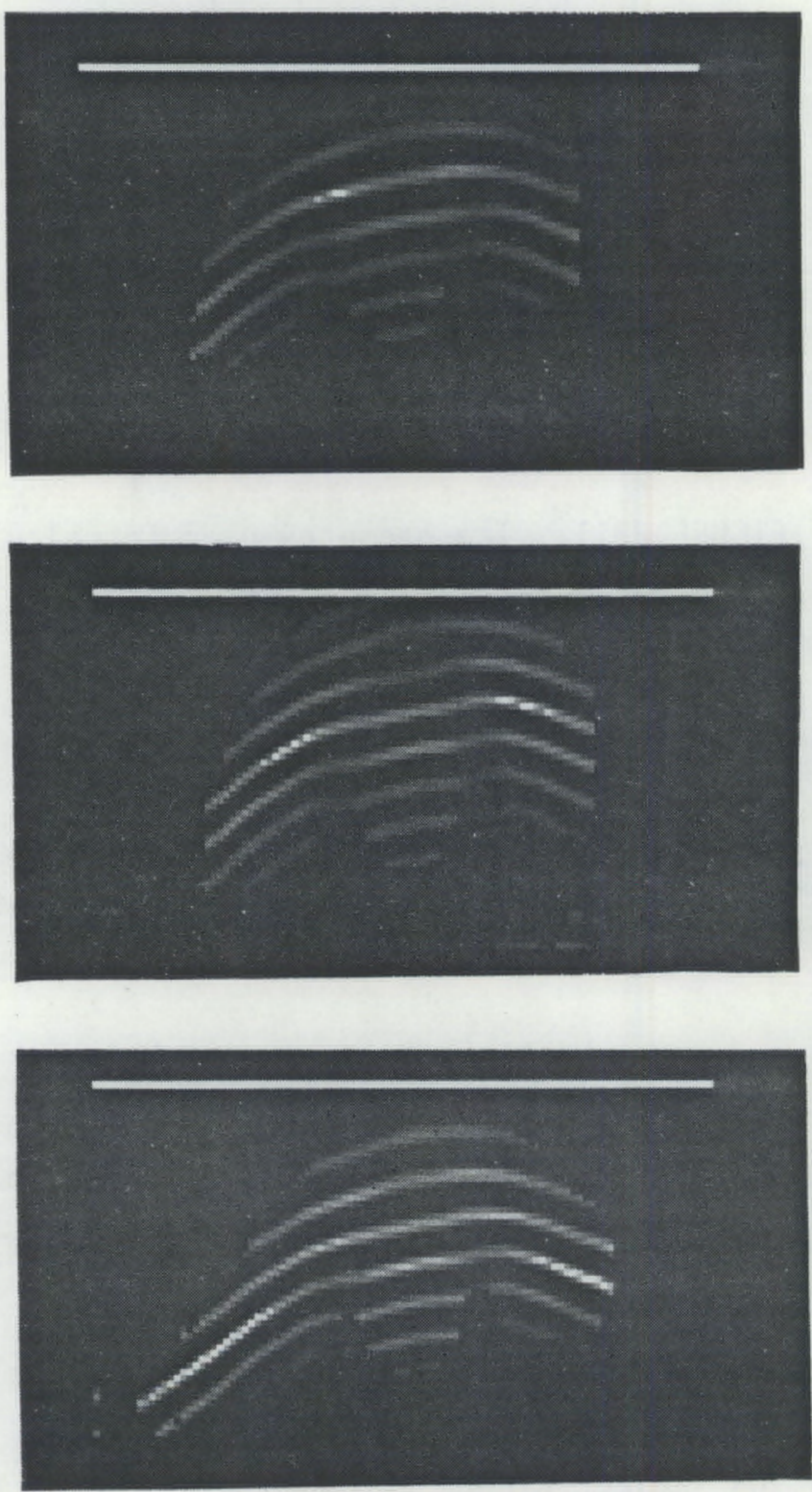

FIGURE 2.10. Correlation data for the simulated overpack canister at a tilt angle of $8.5^{\circ}$. From top to bottom, the images correspond to reference angles of $13.5^{\circ}$, $25.6^{\circ}$, and $35.8^{\circ}$. 


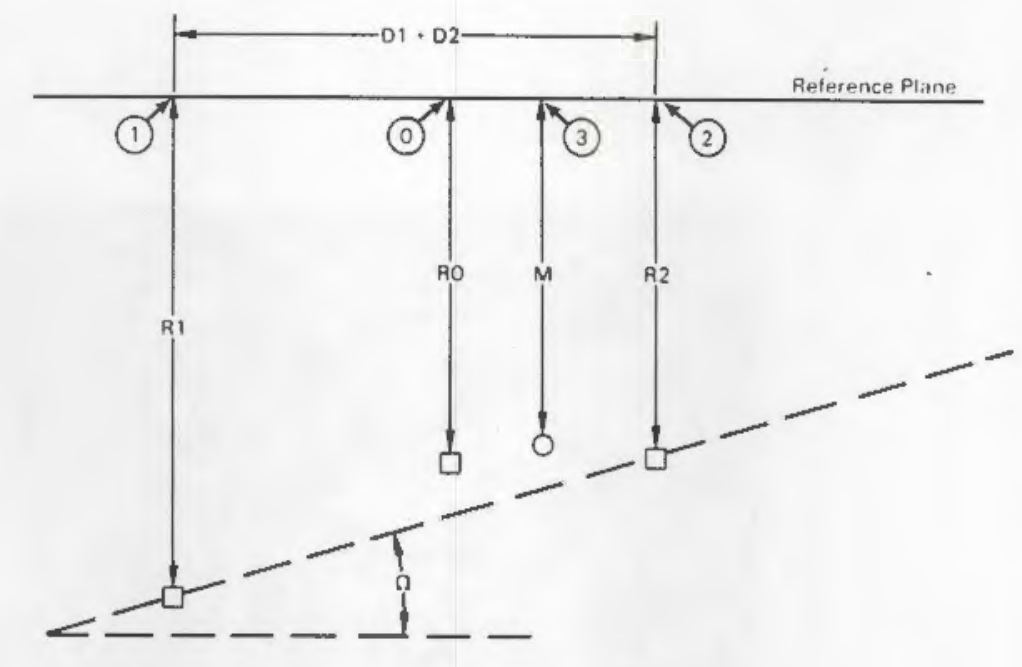

FIGURE 2.11. The geometry used to calculate the canister tilt angle using the correlation data displayed in Figures 2.9 and 2.10.

For angles less than $30^{\circ}$, the difference between $\theta$ and $\Omega$ is less than $2^{\circ}$. (An additional correction was applied to $\Omega$ because the horizontal and vertical scales of the radar profiles do not correspond exactly; the vertical dimension is magnified 1.13 times relative to the horizontal dimension.) The measured tilt angles, converted for measurement geometry and image scale distortion, are as follows:

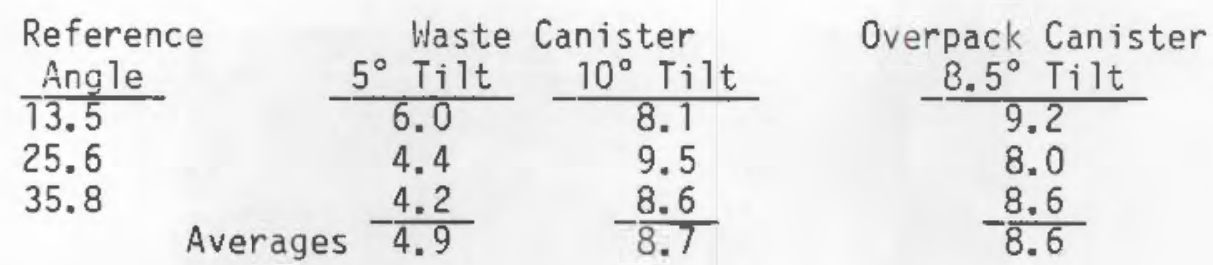

The expected error in these results is approximately $\pm 15 \%$ due to uncertainties in the measurement of along-track distances and difficulty in reading the angles from the photographs. Accuracy is evidentiy better for the larger viewing angles (higher values of $\phi$ ) as expected if errors are introduced by limited precision in position measurements.

The bright spots in the data, especially for large viewing angles, clearly represent patterns of spots rather than unique single points. It would be expected that the backscattered radar waveforms would not change fundamentally over some range of angles, and this is evident in the data. It also appears that this distributed structure in the data may form consistent, characteristic patterns. (Such patterns are common in the angular distribution of radar reflections from complex objects.) If these patterns can be identified reliably, processing techniques similar to those used to relate return waveform 
patterns to viewing angle (i.e., correlation) can be used to improve the resolution of viewing angle determination.

The radar ranges, $R_{1}$ and $R_{2}$, were measured to some external point on the canister (not determined in these tests) rather than to the centerline. The separation of these points complicates the geometry by introducing a conceptual line, representing the effective radar diameter of the structure between the points measured. For small angles, however, for which $\Omega$ and $\theta$ are very nearly the same, this line adds proportionally to both the ranges $R_{1}$ and $R_{2}$ and the separation $D_{1}+D_{2}$ so that there is very little effect on the tilt angle measured.

For actual waste canister measurements in salt, the radar position will be determined with an accuracy of about $1 \mathrm{~cm}$ rather than with errors of up to $15 \mathrm{~cm}$ as in some of these data. A corresponding increase in tilt angle measurement precision is expected. Software will also be developed to determine angles directly from the data with a further anticipated increase in precision.

The final precision of tilt measurements expected using this technique is better tian $1^{\circ}$, perhaps as good as $0.5^{\circ}$. The accuracy of the measurements may be limited by signal refraction near the emplaced canisters. The use of data obtained by viewing at a number of different angles and through different paths provides the potential for identifying refractive variations, which would appear as inconsistencies in the measurements. A complicating factor in these measurements, which may have reduced the accuracy of the results, is that the radar system response is itself dependent on the angular location of the target with respect to the radar antenna axis. The resulting variation in radar return waveform as a function of angle between the radar antenna axis and the target axis was not corrected for in processing the data. The effect is probably sma 11, but should be investigated in further work.

The correlation analysis described above was applied to two-dimensional backscattering data; i.e., the plane in which the canister was tilted was known independently and was not determined from the radar data. In follow-on work, the analysis would be extended to the three-dimensional case. Also, significant additional data may be provided by examining the polarization properties of radar returns from out-of-plane viewing angles. Conventional GPR systems do not provide complete polarization data, but the limited information obtained in these early tests indicates that return polarization is very sensitive to out-of-plane viewing angles. This topic will be explored further in Phase 2 and, if appropriate, the Phase 3 measurement system could be designed to acquire complete polarization data.

\subsection{RADAR SIGNAL PROPAGATION IN SALT AND BENTONITE}

In order to monitor buried waste canisters in the WIPP facility, radar signals may have to be transmitted through several materials. The predominant material is rock salt (halite) of the Permian-age Salado Formation which forms a thick layer at the WIPP site. This material contains minor amounts of clay, polyhalite, anhydrite, gypsum, and other minerals. Another material, not normally associated with the salt but potentially significant with respect to the propagation of radar signals near the buried canisters, is bentonite. This 
clay material is being considered for use in substantial quantities (in the form of a bentonite/sand mixture) as a mechanical buffer and a moisture absorber around the canisters. It may also be used to backfill the emplacement holes after the canisters have been inserted.

In a dry state, none of these material is likely to significantly affect the propagation of the transmitted or reflected radar signals. On the other hand, the velocity, attenuation, and reflection of the radar signals are sensitive to the amount of moisture contained in the materials. An increase in the moisture content of any of these materials, particularly the salt and the bentonite, will result in a reduced propagation velocity, an increased attenuation rate, and an increased reflectivity. Thus, the presence of saturated salt or wet bentonite would be a potential limiting factor on the use of radar to monitor waste canisters. Even a thin Jayer of either of these materials could constitute a substantial absorptive and reflective barrier to the propagation of a radar signal. A brief introduction to the topic of signal propagation in the ground is presented in the Appendix.

\subsubsection{Available Data}

Dry rock salt such as that present at the WIPP site exhibits very low values of electrical conductivity--typically $10^{-5}-10^{-4} \mathrm{Si} / \mathrm{meter}$. Consequently, it is a highly favorable medium for radar signal propagation. Several studies involving radar measurements in salt have been reported ${ }^{1-5}$. The signal attenuation rates experienced in these studies have apparently been less than $0.1 \mathrm{db} / \mathrm{m}$. At this attenuation rate, absorption losses in the salt would be nearly negligible at the short ranges required for canister monitoring.

The radar signal velocity in a typical, natural, rock salt body is approximately $1.2 \times 10^{8} \mathrm{~m} / \mathrm{sec}$. This quantity is determined almost entirely by the dielectric constant of the salt.

In its dry state, bentonite is not a strong absorber of radar signals. However, its radar signal absorption rate increases dramatically with the addition of a small amount of water. A brief literature search conducted for this project did not reveal much experimental data on the electrical properties of this material, although some data for frequencies outside our range of interest is presented in References 7 and 8 . A considerable amount of pertinent data is available for similar clay materials, however.

Tabie 2.1 lists some of the available data relating to the radar signal propagation properties of salt and bentonite. This table is intended only to provide a general overview of the range of possible values for these materials; it is not a definitive compilation of data. Consequently, we have felt free to estimate certain quantities that were not directly available. 
TABLE 2.1. Parameters Relating to Radar Signal Propagation in Selected Materials

\begin{tabular}{|c|c|c|c|c|c|c|c|}
\hline Material & $\begin{array}{l}\text { Frequency } \\
(\mathrm{MHz})\end{array}$ & $\begin{array}{l}\text { Moisture } \\
\text { Content } \\
(w t . \%)\end{array}$ & $\begin{array}{c}\text { Relative } \\
\text { Dielectric } \\
\text { Constant } \\
\end{array}$ & $\begin{array}{c}\text { Signal } \\
\text { Velocity } \\
\left(10^{8} \mathrm{~m} / \mathrm{sec}\right) \\
\end{array}$ & $\begin{array}{l}\text { Conductivity } \\
(\mathrm{Si} / \mathrm{m}) \\
\end{array}$ & $\begin{array}{c}\text { Attenuation } \\
\text { Rate } \\
(\mathrm{dB} / \mathrm{m}) \\
\end{array}$ & Reference \\
\hline Salt & $\begin{array}{l}300 \\
500 \\
440\end{array}$ & $\begin{array}{l}<4 \\
<4 \\
<4\end{array}$ & $\begin{array}{l}-- \\
5.5 \\
6.83\end{array}$ & $\begin{array}{l}-\overline{1} \\
1.28 \\
1.15\end{array}$ & $\begin{array}{c}-- \\
-10^{-4}\end{array}$ & $\stackrel{-.03}{\text { Range }} 23 \mathrm{~m}$ & $\begin{array}{l}1 \\
2 \\
4\end{array}$ \\
\hline $\begin{array}{l}\text { Sait Water } \\
\left(50^{\circ} \mathrm{C}, 180 \mathrm{KPPM}\right.\end{array}$ & $\begin{array}{l}1100 \\
\mathrm{NaCl})\end{array}$ & - & -30 & .55 & -32 & 4300 & 6 \\
\hline Bentonite & $\begin{array}{r}10 \\
4000 \\
4000 \\
4000\end{array}$ & $\begin{array}{r}-- \\
-5 \\
-15 \\
-22\end{array}$ & $\begin{array}{r}4.3 \\
3.8 \\
7.8 \\
12.0\end{array}$ & $\begin{array}{l}1.45 \\
1.54 \\
1.07 \\
.87\end{array}$ & $\begin{array}{l}4.3 \times 10^{-6} \\
.21 \\
.58 \\
1.16\end{array}$ & $\begin{array}{l}176^{.003} \\
346 \\
569\end{array}$ & $\begin{array}{l}7 \\
8 \\
8 \\
8\end{array}$ \\
\hline Clay & $\begin{array}{l}1074 \\
1074 \\
1074\end{array}$ & $\begin{array}{l}10 \\
25 \\
40\end{array}$ & $\begin{array}{r}6.0 \\
9.5 \\
20.0\end{array}$ & $\begin{array}{l}.22 \\
.97 \\
.67\end{array}$ & $\begin{array}{l}.08 \\
.14 \\
.34\end{array}$ & $\begin{array}{r}57 \\
78 \\
127\end{array}$ & $\begin{array}{l}9 \\
9 \\
9\end{array}$ \\
\hline Clay-Loam Soil & $\begin{array}{r}100 \\
1000\end{array}$ & $\begin{array}{l}10 \\
10\end{array}$ & $\begin{array}{l}13.0 \\
10.0\end{array}$ & $\begin{array}{l}.83 \\
.95\end{array}$ & $\begin{array}{l}.04 \\
.14\end{array}$ & $\begin{array}{l}17 \\
71\end{array}$ & $\begin{array}{l}10 \\
10\end{array}$ \\
\hline Sálado Salt & $300-600$ & -2 & 6.35 & 1.19 & - & - & Current Work \\
\hline $\begin{array}{c}\text { Bentonite/Sand } \\
(70 \% / 3 C \%)\end{array}$ & $\begin{array}{l}300-600 \\
300-600\end{array}$ & $\begin{array}{l}10 \\
12\end{array}$ & $\begin{array}{l}5.4 \\
5.4\end{array}$ & $\begin{array}{l}1.29 \\
1.29\end{array}$ & $\begin{array}{l}.043 \\
.051\end{array}$ & $\begin{array}{l}37 \\
37\end{array}$ & $\begin{array}{l}\text { Current Work } \\
\text { Current Work }\end{array}$ \\
\hline
\end{tabular}

\subsubsection{Measurements on Salt and Bentonite}

The last three entries in Table 2.1 represent measured data obtained during this project. Measurements of the signal propagation velocity and attenuation rate in sait and bentonite were not included in the original plan for this Phase 1 work; however, as the project developed, it seemed appropriate to include modest efforts in these areas. The measurements were performed in a simple, "quick and dirty" manner using the available GSSI radar unit. The results should be regarded as both approximate and preliminary.

The signal velocity in salt was measured in a cylindrical salt block extracted from the floor of the WIPP facility. Minor amounts of clay were distributed throughout the block, mainly as thin, irregular patches between halite crystals. Other minerals associated with the halite were presumably disseminated uniformly throughout the volume. No macroscopic layers or fractures were visible.

The block was $86.4 \mathrm{~cm}$ in diameter and $58 \mathrm{~cm}$ long. A drilled hole $5 \mathrm{~cm}$ in diameter passed through the length of the block on the cylinder axis.

The radar signal velocity was measured both verticaliy (between the ends of the cylinder) and horizontally (across the diameter). In the former case, the $900-\mathrm{MHz}$ GSSI antenna was placed on the top of the block. Signals reflected from the bottom of the block were recorded and analyzed to determine their round-trip travel time. In the latter case, the antenna was mounted against the side of the block. The recorded signals included reflections from: 1) the open center hole, 2) an aluminum rod in the center hole, and 3) an 
aluminum rod held against the side of the block at a point diametrically opposite the antenna. The measured travel times together with the dimensions of the block gave the velocity listed in Table 2.1. The signal attenuation was too small to estimate in this simple experimental arrangement.

Measurements of signai velocity and attenuation in bentonite were made in a similar manner. A wooden box $91 \mathrm{~cm}$ square by $38 \mathrm{~cm}$ high was filled to a depth of $38 \mathrm{~cm}$ with a mixture consisting of $70 \%$ (wt) granular bentonite and $30 \%$ fine-grained quartz sand. The initial moisture content of the mixture was $10.2 \%$ by weight as determined by weighing a sample before and after heating at a temperature of $150-160^{\circ} \mathrm{C}$ for 34 hours.

The $900-\mathrm{MHz}$ antenna was mounted on one side of the box. A thin metal plate, oriented such that its surface was paraliel to the front surface of the antenna, was inserted vertically into the bentonite/sand mixture and the signals reflected from this plate were recorded. Measurements of this type were made at seven antenna-target distances ranging from 25 to $60 \mathrm{~cm}$. After this first series of measurements was completed, water was added to the mixture (with thorough mixing) and the measurements were repeated. The measured moisture content for this second set of measurements was $12.2 \%$.

The signal velocity given in Table 2.1 was determined from a plot of antenna-target distance vs. travel time. The attenuation rates were estimated by fitting the measured amplitude vs. distance values by functions of the form

$$
f(d)=\frac{C e^{-2 \alpha d}}{d^{p}}
$$

where $C$ is a constant, $d$ is the antenna-target separation, $P$ is an exponent related to the geometrical spreading of the signal, and $a$ is the attenuation constant in units of nepers/m (see Appendix). The attenuation rate $A$ is related to $a$ by $A=8.69 \alpha \mathrm{dB} / \mathrm{m}$.

\subsubsection{Reflection and Refraction}

The backscattering measurements described in Section 2.1 indicate that the location and orientation of the waste canisters can be accurately determined by the radar method provided that the canisters are contained in a uniform dielectric medium (e.g., air or salt). However, the actual signal propagation medium may be non-uniform due to the presence of bentonite or crushed salt in the burial hole and the possible localization of moisture in these materials.

At an interface between any two materials, the radar signal will undergo both refraction and partial reflection. Refraction (bending of the signal path) is likely to be the more important factor in terms of its effect on measurement accuracy. Partial reflections will reduce signal amplitude, but should not be a serious problem unless the reflection coefficient approaches unity at a critical interface.

Figure 2.12 shows three possible ray paths for signals backscattered from the ends of a canister. The ray backscattered from the top of the canister 
illustrates refraction of the salt-bentonite interface for a case where the signa) velocity is greater in the salt than in the bentonite (as indicated in Table 2.1, the reverse may be true when the bentonite is dry). The figure also illustrates a case where the containment hole is on ly partially filled with the bentonite/sand mixture.

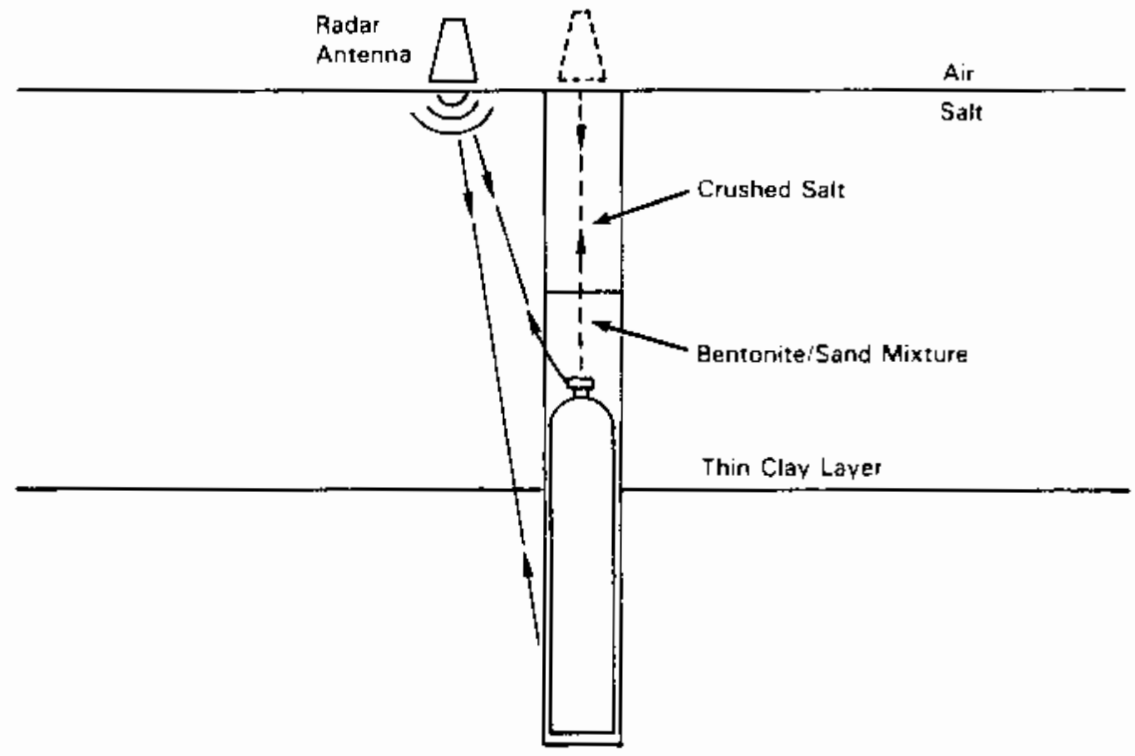

Figure 2.12. Examples of ray paths for radar signals backscattered from a canister buried in salt with bentonite/sand backfill material. A possible thin clay layer is also illustrated.

17though the geometry shown in Figure 2.12 offers several possibilities for refraction, it represents a highly symmetrical situation in which the difficulty of measuring the location and orientation of the canister would be at a ninimurn. Acditional sources of inaccuracy would be present if one or more of the material interfaces were tilted. Some tilting is expected to occur, of course, given enough time for the salt to flow in response to pressure gradients. A significant refractive effect may also occur at the air-salt interface (i.e., at the floor of the storage room) if that surface is not quite horizontal. Fortunately, it should be possible to determine interface tilts from the reflected radar signals or, in the case of the floor surface, by direct measurement. The effects of refraction may thus be computed and corrected for if the properties of the materials involved are known.

The magnitude of the corrections needed to determine canister location and tilt can be easily estimated if we assume a nearly vertical viewing angle. Figure 2.13 shows a simplified radar propagation path from the surface through layers of salt and bentonite to a waste canister. The path shown is the one that arrives along the canister's axis. The interfaces between various layers are shown inclined at angles $\theta_{1}$ for air to salt and $\theta_{2}$ for salt to bentonite. The canister tilt angle with respect to vertical is $\theta_{3}$. 


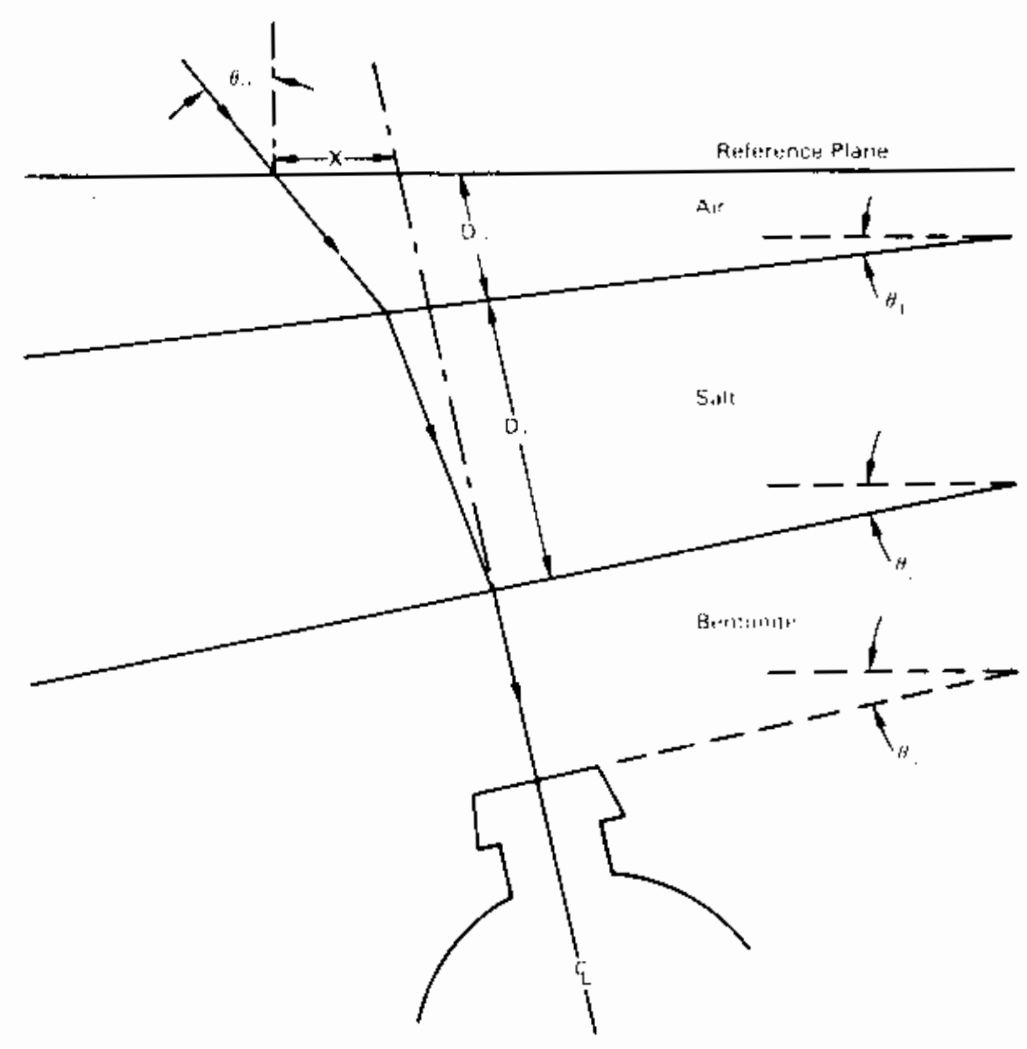

FIGURE 2.13. Refraction of a radar signal specularly reflected from the canister pintle.

The angle $\theta_{0}$ at which the refracted, or apparent, centerline appears at the surface will not, in general, be the same as $\theta_{3}$. As shown, the refracted centerline will also be displaced by some distance $x$ from the true extended centerline. The angle $\theta_{0}$ and the displacenent $X$ are approxinated by

$$
\theta_{0} \cong \theta_{1}+N_{s}\left(\theta_{2}-\theta_{1}\right)+N_{b}\left(\theta_{3}-\theta_{2}\right)
$$

and

$$
X \cong D_{0}\left[\theta_{1}+N_{s}\left(\theta_{2}-\theta_{1}\right)+H_{b}\left(\theta_{3}-\theta_{2}\right)\right]+D_{1} N_{b}\left(\theta_{3}-\theta_{2}\right)
$$

Where $N_{s}$ and $N_{b}$ are the radar indices of refraction for salt and bentonite, respectively. (The index of refraction is the factor by which radar propagation velocity is reduced in the material relative to air. The numerical values would be about 2.5 for both salt and bentonite.) The above equations were derived by making the approximations, valid for small angles (in radians), that

$$
\sin \phi=\tan \phi=\phi \text {. }
$$

Flaking the additional simplifying assumption that $M_{b}$ is equal to $N_{s}$, which is equivalent to reducing the situation to a single layer of index $H$, we obtain 


$$
\theta_{0} \cong(1-N) \theta_{1}+N \theta_{3}
$$

and

$$
X \cong D_{0}\left[(N-1) \theta_{1}+N \theta_{3} j+D_{1}\left(\theta_{3}-\theta_{1}\right)\right.
$$

The effect of the salt layer on the perceived canister tilt angle is thus to magnify the actual canister tilt by a factor of approximately $\mathrm{N}$ (numerical value 2.5) and introduce a correction of $1-\mathrm{N}$ (numerical value 1.5) times the tilt of the air-salt interface. As would be expected for a single-layer model, the spatial displacement of the perceived centerline depends on the (magnified) tilt angle and the height of the reference plane above the salt surface.

Apparentiy, the effect of refraction at layer boundaries will be significant and must be computed and corrected for in data analysis. The most significant refraction is expected at the airasalt boundary, but the tilt of this surface relative to a horizontal reference plane can be directly measured. As suggested above, the tilt angles of subsurface interfaces can probably be determined from the reflected radar signals. Non-planar interfaces will complicate the correction process.

A situation not covered by the simple model of distinct, homogeneous layers is the case of continuous velocity gradients. Such gradients, if present, would produce only a smali amount of refractive bending, but data are not available. The use of radar measurements obtained through a number of different paths to the canister may allow the detection of errors in the refractive correction. Uncompensated refractive errors would cause the measurements to disagree. The occurrence and magnitude of discrepancies would thus indicate the existence and magnitude of otherwise undetected refractive effects.

During operation of a radar sensing system, one or more antennas would be moved along a regular, predetermined, grid pattern located a few centimeters above the floor of a canister storage room. Signals traveling between the antennas and a buried canister will follow refracted paths as indicated in Figures 2.12 and 2.13. Each refractive interface along a given path will attenuate the signal by reflecting part of its energy in an undesired direcion. These losses, which occur equally on each leg of the two-way signal path, could significantly reduce the amplitude of the received signal. If the signal amplitude were reduced to a level comparable to that of the random noise present in the radar receiver, the accuracy of the measurement would be severely degraded. The point at which the radar method would become ineffective would be determined by the power and sensitivity of the radar unit and by the signal reflection coefficients at the material interface.

The reflection of an electromagnetic wave at a planar interface is discussed in the Appendix. It is shown there that the reflection coefficient at a given interface is determined by the dielectric constants and electrical conductivities of the materials. The equations given in the Appendix, together with data from Table 2.1. give the following values for the magnitude of the complex amplitude reflection coefficient at four possible material interfaces: 


\begin{tabular}{ll}
\multicolumn{1}{c}{ Interface } & $|R|$ \\
\cline { 3 - 3 } Air-Salt & \\
Air-Bentonite & .43 \\
Salt-Bentonite & .42 \\
Salt-Brine & .09 \\
& .85
\end{tabular}

These values were calculated for signals that are vertically incident on the interfaces and would be higher for obliquely incident signals. Nevertheless, they show that a salt-bentonite interface is relatively benign at moderate levels of bentonite moisture content ( $12 \%$ was assumed as in Table 2.1$)$. A salt-brine interface, on the other hand, would be a strong reflector of radar energy. In view of the fact that the dynamic range of the radar unit will almost certainly be greater than $100 \mathrm{~dB}$ (a range of $10^{5}$ in signal amplitude), these reflection coefficients do not seem to represent a serious problem except in combination with the strong attenuation rates expected for brine and moist bentonite. 


\subsection{GPR HARDWARE}

\section{1 DISCUSSION}

The ground-penetrating radar system used to collect backscattering data in this feasibility study is not necessarily the optimum system to use in the planned operational monitoring system. The GSSI radar unit was designed to perform a broad range of subsurface sensing tasks (e.g., pipe detection, chemical waste mapping, rebar detection in concrete) under widely varying environmental conditions. Thus, it is unlikely to represent an optimum system for the very specific task of monitoring waste canisters in salt. The main design factors to be addressed in defining an optimum system for the WIPP application are:

- transmitter power

- frequency spectrum of the transmitted signal

- system bandwidth

- antenna beamwidth

- antenna impedance (matching to salt)

- antenna polarization.

These factors are discussed very briefly in the following paragraphs.

Transmitter Power--The average power transmitted by the GSSI Model 1010 antenna used in this study was approximately $0.06 \mathrm{~mW}$. Increasing this power ievel by increasing the amplitude of the transmitted pulse or by increasing the pulse repetition rate would improve the effective signal-to-noise ratio of the received signals.

Signal Noise--The random electronic noise present at the output of the radar receiver circuit can be minimized by careful circuit design. A critical point is to amplify the received signals to a level of approximately $0.1 \mathrm{~V}$ prior to the gating operation needed to sample and digitize the high-frequency signals.

Frequency Spectrum--In order to achieve the desired accuracy in measurements of canister location and tilt, it seems necessary to utilize short (approximately 1-nsec long) radar impulses. Such impulses correspond to broad frequency spectra which span several octaves around a center frequency of approximately T $\mathrm{GHz}$.

System Bandwidth--A short (broad spectrum) radar signal can be transmitted and received without distortion only if all components of the radar system have a wide spectral bandwidth. The GSSI antennas, and most others used in GPR applicatons, are inherently narrow-band devices which distort and lengthen the transmitted radar signals. For high-resolution measurements, it may be desirable to utilize antennas of an alternative design. In addition, the receiver electronics must have a bandwidth consistent with that of the antennas.

Antenna Beamwidth--An additional aspect of antenna design in this application is the need to illuminate a target canister over a wide range of viewing angles. A beamwidth of approximately $\pm 45^{\circ}$ at the half-power points is needed to maintain an acceptable signal amplitude over the entire angular range. 
Antenna Impedance--Unwanted reflections of the radar signals at the apertures of the transmitting and receiving antennas can be minimized by matching the aperture impedance of the antennas to that of the external medium. In this case, the appropriate medium is salt and the impedance to be matched is approximately 150 ohms (see Appendix).

Antenna Polarization--The backscattering measurements made in this study indicate that the characteristics of signals backscattered from a tilted canister are dependent upon the polarization of the transmitted signal. Although this effect is not discussed in detail in this report. it is significant and should be considered further in subsequent work. It may be possible to utilize polarization data to determine canister orientation.

\subsection{PRODUCT SURVEY}

A survey of commercially available GPR systems was conducted in order to determine whether a radar unit suitable for canister monitoring could be obtained "off the shelf." Six companies involved in the development and sale (or use) of GPR systems were contacted. These were:

1) Geophysical Survey Systems, Inc. (GSSI)

15 Flagstone Drive

Hudson, New Hampshire 03051

2) MACOM

Burlington, Massachusetts

3) XADAR Corporation

5400 Port Royal Road

P.0. Box 1162

Springfield, Virginia 22151

4) OYo Corporation (USA)

7334 N. Gessner Road

Houston. Texas 77040

5) Penetradar Corporation

6865 Walmore Road

Niagra Falis, New York 14304

6) Gulf Applied Research

Suite $S$

2260 Northwest Parkway, S.E.

Marietta, Georgia 30067

As one might expect, these companies provided some general system design information, but not much detailed information regarding antenna design, pulse generator design, and so on. The configurations of the GPR systems produced by four of these companies are illustrated in Figures 3.1-3.3. 

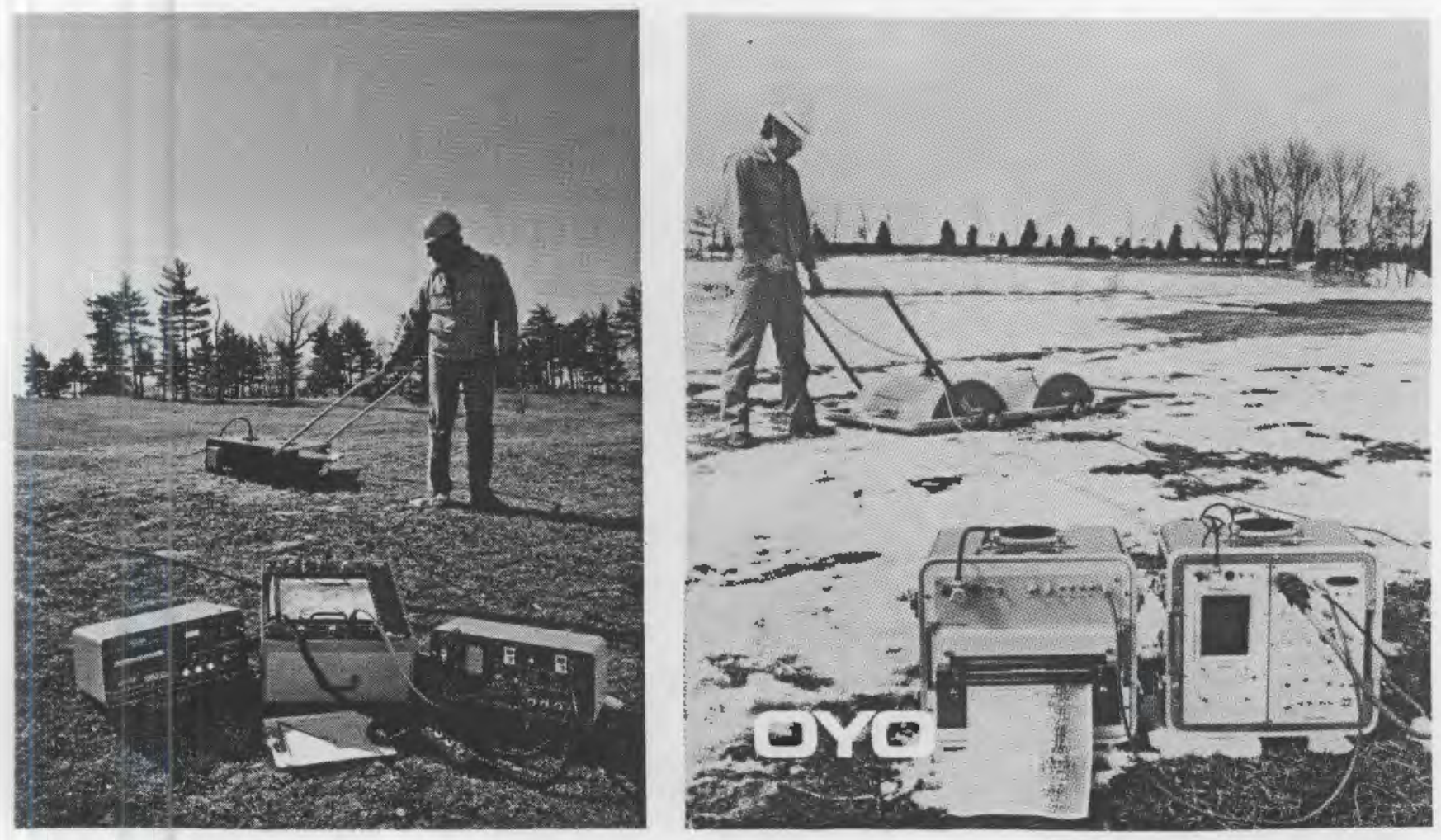

FIGURE 3.1. GPR systems produced by GSSI (left) and 0 YO.

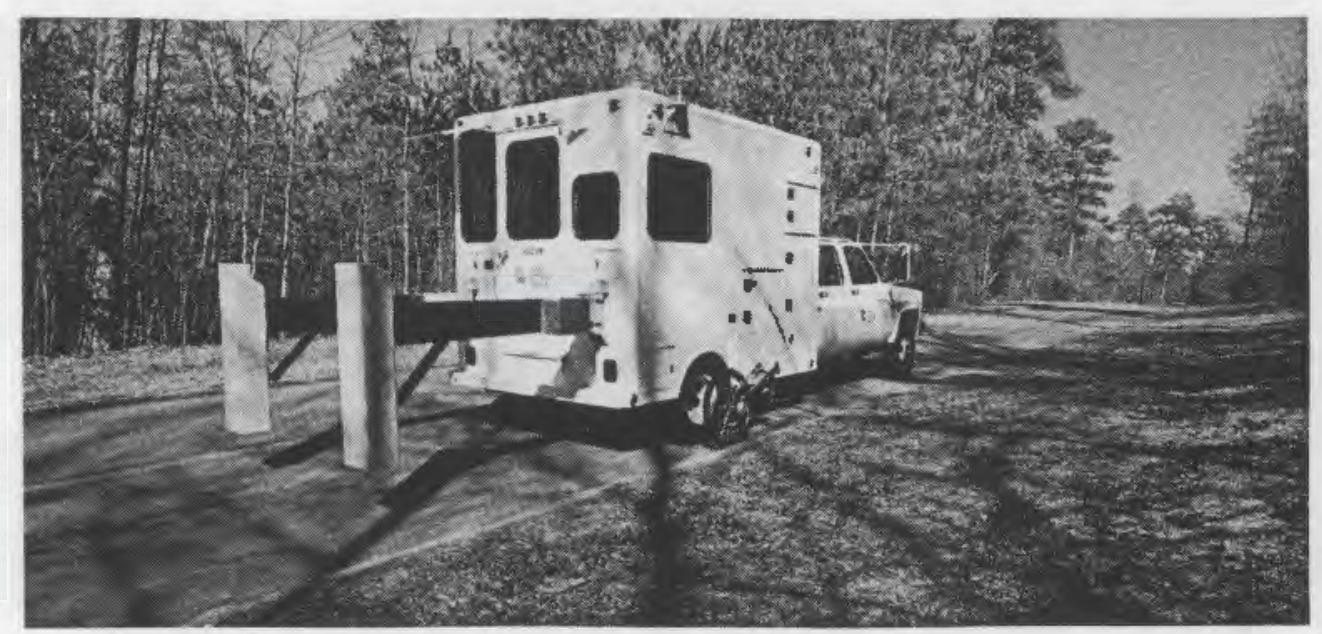

FIGURE 3.2. Gulf Applied Research system for highway inspection. 


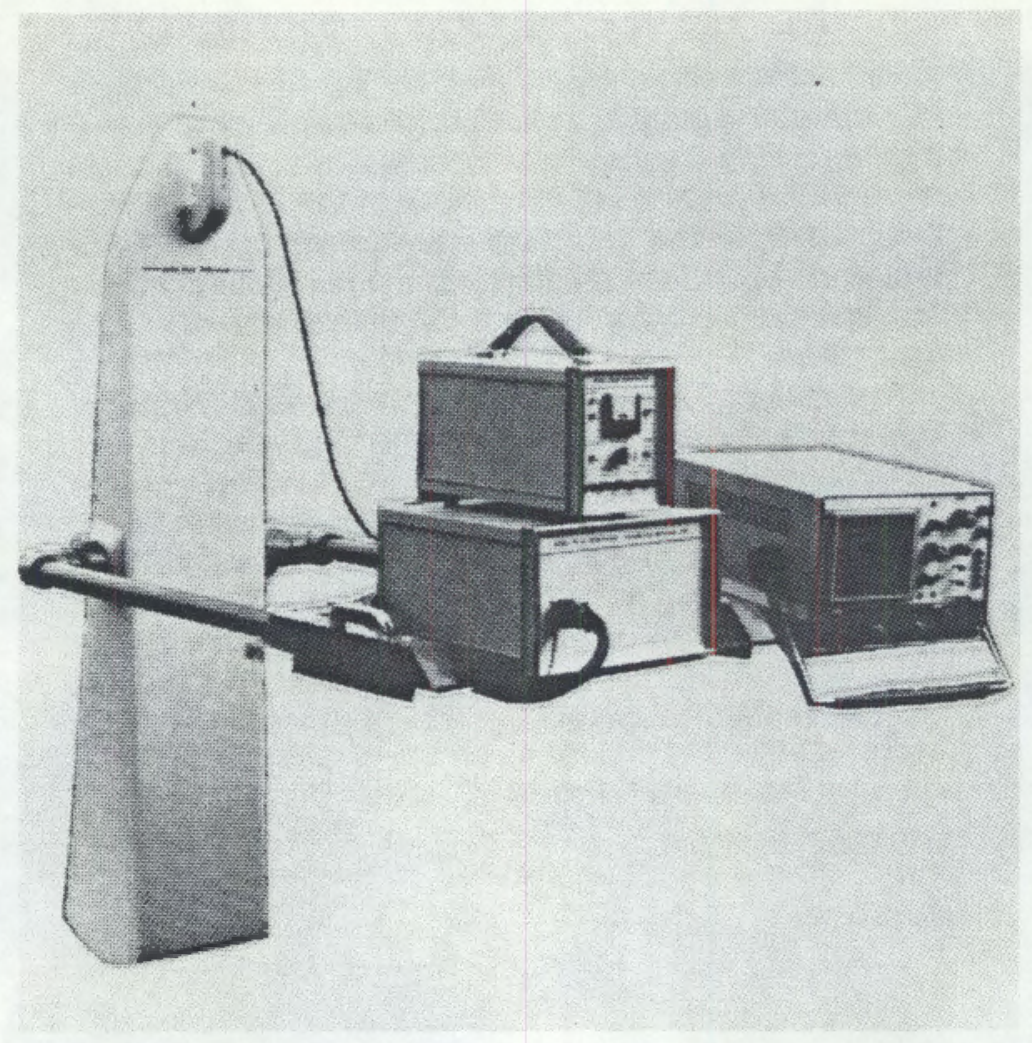

FIGURE 3.3. The Penetradar GPR system.

Table 3.1 summarizes the information obtained from the companies listed above. Note that two distinct systems are described for both XADAR and Gulf Applied Research. It is also significant that all but one of the GPR systems listed are impulse-type systems operating on the principle of time-domain reflectometry. The exception, the synthetic pulse concept under development by XADAR, is an interesting approach that may yield improved penetration depths. Its main limitations appear to be its slowness of operation and its relatively poor spatial resolution. A number of other innovative and potentially useful design concepts are represented in this list; for example, the monostatic antenna configuration used by Penetradar, the AGC functions employed by XADAR and OYO, the $1-\mathrm{MHz}$ pulse repetition rate used by Penetradar, and the raised antenna being investigated by Gulf Applied Research.

In summary, however, none of these systems represents an ideal design for the unique application being studied in this project. In particular, the antenna used in these systems do not provide the desired combination of bandwidth, beamwidth, and impedance matching as described in Section 3.1. An appropriate radar unit for this application would probably consist of a specially designed antenna interfaced to a basic electronics package similar to those developed for the commercial products. 
TABLE 3.1. Summary of the Characteristics of Commercial GPR Systems

\begin{tabular}{|c|c|c|c|c|c|c|c|c|}
\hline & Syster & & $\begin{array}{l}\text { Principal } \\
\text { Application }\end{array}$ & $\begin{array}{c}\text { Unique } \\
\text { Features }\end{array}$ & $\begin{array}{l}\text { Antenná } \\
\text { Irpe }\end{array}$ & $\begin{array}{l}\text { Output Power, } \\
\text { Pulse Length }\end{array}$ & $\begin{array}{l}\text { Frequency } \\
\text { Range }\end{array}$ & Cumnents \\
\hline & GSS] & & General purpose & $\begin{array}{l}\text { First colluercial } \\
\text { systemi }\end{array}$ & Bow tie & $\frac{<42 \text { W peak }}{1-6 \text { nsec }}$ & $\begin{array}{l}50-1200 \mathrm{MHz} \\
\text { vid interchangeable } \\
\text { transce fyers. }\end{array}$ & Widely used, flexible systenk. \\
\hline & $X A G A R$ & 1) & General purpose & $\begin{array}{l}\text { Hicroprocessor-based } \\
\text { contrai unit. } \\
\text { Digital IVG \& AGC }\end{array}$ & $\begin{array}{l}\text { Bow tie } \\
\text { Bistatic } \\
\text { conf \{guration }\end{array}$ & $\begin{array}{l}50-250 \text { w pead } \\
\text { i } 1-6 \text { nsec }\end{array}$ & $\begin{array}{l}50-1200 \text { Mltz est. } \\
\text { vid interchangeable } \\
\text { transceivers. }\end{array}$ & $\begin{array}{l}\text { Similor to GSS1, more expen- } \\
\text { sive, nlaybe better control } \\
\text { unit. }\end{array}$ \\
\hline & & 2) & Hining surveys & $\begin{array}{l}\text { Syuthetic puise } \\
\text { systern }\end{array}$ & Unknown & Uniknown & $200-160 \mathrm{HHz}$ & $\begin{array}{l}\text { System under development. } \\
\text { Slow operation due to frequency } \\
\text { steps. Good depth penetration. } \\
\text { Not. to be marketed. }\end{array}$ \\
\hline & Penetrad & & $\begin{array}{l}\text { Highway } \\
\text { inspection }\end{array}$ & $\begin{array}{l}\text { Single-antenna } \\
\text { design. } 1 \text { - Hlliz pulse } \\
\text { rate. }\end{array}$ & $\begin{array}{l}\text { Wedge TEN Horn } \\
\text { (THET \} }\end{array}$ & $.75-2.0$ nsec & $=100-2500 \mathrm{Hkz}$ & $\begin{array}{l}\text { Low power system intended for } \\
\text { very shallow penetration. } \\
\text { Origindlly developed to detect } \\
\text { land mines. }\end{array}$ \\
\hline & $\begin{array}{l}\text { Gulf } \\
\text { Applied } \\
\text { Research }\end{array}$ & 1) & $\begin{array}{l}\text { Highway } \\
\text { inspection }\end{array}$ & $\begin{array}{l}\text { Total package } \\
\text { includes survey truck. }\end{array}$ & Weḍ̣e TEH Horn & $\begin{array}{l}20 \mathrm{~W} \text { peak } \\
\text { est. }\end{array}$ & $200-2000 \mathrm{MHz}$ & System not narketed. \\
\hline & & 2) & $\begin{array}{l}\text { General } \\
\text { purpose }\end{array}$ & $\begin{array}{l}\text { High power. } \\
\text { Raised antenna } \\
\text { planned }\end{array}$ & $\begin{array}{l}\text { Bow tie } \\
\text { 0thers tested }\end{array}$ & $\begin{array}{l}>2000 \mathrm{~W} \text { peak } \\
3 \mathrm{nsec}\end{array}$ & $\because 150-450 \mathrm{NHz}$ & $\begin{array}{l}\text { Sys ten under development. } \\
34 \text { dB improvenent over fiss I } \\
\text { unit. }\end{array}$ \\
\hline & aro & & $\begin{array}{l}\text { Generol } \\
\text { purpose }\end{array}$ & $\begin{array}{l}\text { Selectable preamp } \\
\text { gain. AGC. Color } \\
\text { display. }\end{array}$ & $\begin{array}{l}\text { Bow tie? } \\
\text { gistatic } \\
\text { configuration }\end{array}$ & $\begin{array}{l}270 \text { valts } \\
8 \mathrm{nsec}\end{array}$ & $60-180 \mathrm{HHz}$ est. & Several innovative features. \\
\hline & MACON & & $\begin{array}{l}\text { Pipe \& cable } \\
\text { detection }\end{array}$ & $\begin{array}{l}\text { Locator, not illapper. } \\
\text { Stepwise theasure- } \\
\text { ment itode. }\end{array}$ & $\begin{array}{l}\text { Floppy dipoles } \\
\text { (crossed) }\end{array}$ & Isnknown & $30-300 \mathrm{kitz}$ & $\begin{array}{l}\text { Terrascan system (onio state } \\
\text { University). In redesignt } \\
\text { phase. }\end{array}$ \\
\hline
\end{tabular}




\subsection{LITERATURE SEARCH}

R\&D efforts in the fields of radar and RF communication over the past several decades have resulted in the publication of thousands of papers on antenna design. Although very few of these papers directly address the design of GPR antennas per se, some of them describe antennas that can be adapted to GPR usage. The objective of this literature search was to acquire some of the key recently published papers relating to existing GPR antenna designs. Our approach included searches of the following computer data bases:

- NTIS - The National Technical Information Service listing of government reports in a broad range of technical fields.

- Engineering Index - A comprehensive listing of engineering-oriented papers published in international technical journals and books.

- INSPEC - A comprehensive international listing of papers, reports, and books covering the fields of physics, electrical engineering, and computers.

Additional publications available in the technical library of the Pacific Northwest Laboratory were examined as a supplement to this computer search. The most useful of these were the IRE and IEEE Transactions on Antennas and Propagation. The Bibliography at the end of this report lists some of the reports, papers, and books that were acquired and examined as a resuTt of this literature search.

Table 3.2 summarizes the principal operational characteristics of four basic antenna types which have proven to be effective in GPR applications. The quoted values of geometric gain are referenced to a theoretical isotropic radiator ( $\mathrm{dBi}$ ). This value can be related to actual signal strengths or sensitivities by muttiplying by antenna efficiency, which, for GPR antenna systems optimized for pulse response and low ringing, will probably be less than $50 \%$ $(-3 \mathrm{~dB})$. All of the values given in this table are estimates or are representative of one particular case; a wide range of variation is possible.

The various dipole antenna designs offer the advantage of a wide beamidth but suffer from an inherently narrow bandwidth. A planar spiral antenna is typically light and compact (an advantage in this application), but exhibits severe phase dispersion. This latter characteristic results in pulse distortion and a loss of spatial information.

The antennas that appear to offer the best performance are three-dimensional structures generally classified as TEM antennas. These antennas may take several forms, but all approximate a tapered transmission line impedance transformer. An antenna of this kind could be enhanced by dielectric loading to reduce its physical size. As far as we know, only the exponentially flared TEM antenna has been tested in a dielectric-filled form. The more recently developed constant-angle wedge antennas offer potential advantages, particularly in reduced ringing, but would require some development to incorporate dielectric loading. 
If future analysis indicates significant advantages in a system with multiple polarization capability, a feasible course of action would be to develop a version of the transmission line wedge antenna that would incorporate orthogonal polarizations in a common volume.

\section{TABLE 3.2. Summary of antenna characteristics}

\begin{tabular}{|c|c|c|c|c|}
\hline \multirow[b]{2}{*}{ CHARACTEEISTIC } & \multicolumn{3}{|c|}{ ANTEHHA TYPES } & \multirow[b]{2}{*}{$\begin{array}{l}\text { Constant Angie, or } \\
\text { Wedge TEM Horn } \\
\text { (GWIA or ThITi }\end{array}$} \\
\hline & $\begin{array}{l}\text { Dipoles, Inclusting } \\
\text { Folded and Bow Tíg } \\
\text { Tyges } \\
\end{array}$ & $\begin{array}{c}\text { Cavity-Backed } \\
\text { Spiral }\end{array}$ & $\begin{array}{l}\text { Exponential and } \\
\text { Ridged Horns }\end{array}$ & \\
\hline Geometric Gain & $\begin{array}{l}\text { थ-5 dBi if radiation } \\
\text { is conf thed to } 1 \text { hemi- } \\
\text { sphere by shieldirig or } \\
\text { ground refraction. }\end{array}$ & $\begin{array}{l}27 \mathrm{dBi} \text {. Actual gain } \\
\text { varies yith frequency } \\
\text { and system efficiency. } \\
\text { Values of }-4 \text { to } 2 \text { dBi } \\
\text { are typical. }\end{array}$ & $\begin{array}{l}5-15 \alpha \mathrm{B} i \text {, typically } \\
\text { frequency dependent. }\end{array}$ & $\begin{array}{l}\text { Frequency dependent. } \\
\text { Typically }-20 \mathrm{CBi} \text { at } \\
\text { lowest useful frecuency } \\
\text { to }+5 \mathrm{~dB} \text { at highest } \\
\text { frequency. Gain is in- } \\
\text { versely proportional to } \\
\text { wavelength (constant } \\
\text { effective aperture?. }\end{array}$ \\
\hline Beamwidth (in air) & $\begin{array}{l}60^{\circ}-90^{\circ} \text { in plane of } \\
\text { electrical axfs. Less } \\
\text { than } 130^{\circ} \text { in plane per- } \\
\text { pendickiar to axis. }\end{array}$ & $70^{\circ}-90^{\circ}$ & $25^{\circ}-75^{\circ}$ & $\begin{array}{l}35^{\circ}-45^{\circ} \text {. Can be } \\
\text { increased by ddiusting } \\
\text { flare angle. }\end{array}$ \\
\hline Bandwidth & $\begin{array}{l}\text { Typically less than } 2: 1 \text {, } \\
\text { but may be widened if } \\
\text { low radiation efficiency } \\
\text { is acceptable. }\end{array}$ & $\begin{array}{l}\text { More than 10:1 } \\
\text { possible. }\end{array}$ & $\begin{array}{l}4: 1 \text { to } 10: 1 \text { or more } \\
\text { depending on details of } \\
\text { design. }\end{array}$ & 20:! or greater. \\
\hline Pulse Distortion & $\begin{array}{l}\text { Eependent on bandwidth } \\
\text { (may depend on ground } \\
\text { coupling). }\end{array}$ & $\begin{array}{l}\text { Distortion produced by } \\
\text { dispersion inherent in } \\
\text { delay line structure. }\end{array}$ & $\begin{array}{l}\text { S1ight. Rolatively low } \\
\text { dispersion. }\end{array}$ & $\begin{array}{l}\text { Lowest distortion of any } \\
\text { kncwn antennd. close } \\
\text { to theoretical millimum. }\end{array}$ \\
\hline Feed Impe Jance & $\begin{array}{l}70 \text { ohms for thin reso- } \\
\text { nant dipole. More than } \\
300 \text { ohms for folded } \\
\text { types. }\end{array}$ & $\begin{array}{l}2200 \text { ohms. Usually con- } \\
\text { verted to } 50 \text { ohms by a } \\
\text { balanced transformer. }\end{array}$ & $\begin{array}{l}\text { Usually made to match } \\
50 \text {-chm transmission } \\
\text { line. }\end{array}$ & Can be made to 50 unms. \\
\hline Polärization & Linear. & $\begin{array}{l}\text { Circular (produced by } \\
\text { delay in the soiral). }\end{array}$ & $\begin{array}{l}\text { Linear. Ridged horns } \\
\text { can have two orthogonal } \\
\text { antennas in common } \\
\text { rolume. }\end{array}$ & Linear. \\
\hline Size & $\begin{array}{l}\text { Length approximately } 1 / 2 \\
\text { wavelength of lowest } \\
\text { frequency. Height and } \\
\text { width dependent on re- } \\
\text { flectors and shieiding. }\end{array}$ & $\begin{array}{l}\text { Diane ter approximately } \\
1 / 3 \text { to } 1 / 2 \text { wayelength } \\
\text { of lowest frequency. }\end{array}$ & $\begin{array}{l}\text { Max. dimensions are } \\
\text { w/2-1 wavelength of } \\
\text { lowest frequency. oi- } \\
\text { electric filler needed } \\
\text { for frequencies as low } \\
\text { as } 50 \text { MHz. }\end{array}$ & $\begin{array}{l}\text { Long axis (height) wl/2 } \\
\text { wavelength of iowest } \\
\text { useful frecuency. }\end{array}$ \\
\hline Nates & $\begin{array}{l}\text { Damping required for } \\
\text { acceptable pulse re- } \\
\text { sponse may make antenna } \\
\text { tnefficient, yielding } \\
\text { gain less than } 0 \text { tBi. }\end{array}$ & & $\begin{array}{l}\text { A broad range of hybrid } \\
\text { waveguide-transmission } \\
\text { line structures using } \\
\text { exponentially increasing } \\
\text { spacing between elenents } \\
\text { to dporaximate a wide } \\
\text { bandwidth impedance } \\
\text { match from a transmis- } \\
\text { sian line to free space. }\end{array}$ & $\begin{array}{l}\text { Developed to transmit } \\
\text { short impulses, so not } \\
\text { characterized by the } \\
\text { continuous wave, single } \\
\text { frequency measurements } \\
\text { commonly used for otner } \\
\text { antenlnas. }\end{array}$ \\
\hline
\end{tabular}




\subsection{CONCLUSIONS AND RECOMMENDATIONS}

\subsection{CONCLUSIONS}

This Phase 1 study was an initial effort to assess GPR technology as a possible means of monitoring buried waste canisters. The principal conclusion to be driwn from the work described on the preceding pages of this report is that the radar method can be effectively used for that purpose.

The backscattering measurements made in the laboratory showed that canister-shaped targets produce strong, easily detectable, backscattered signals from the pintle and shoulder areas of the waste and overpack canisters.

Three data anaiysis methods of varying degrees of complexity were shown to produce good estimates of the location and tilt of the canister axis. The accuracy of the results obtained was within the tolerances prescribed as system performance goals ( $\pm 5 \mathrm{~cm}$ in position, $\pm 2^{\circ}$ in tilt angle). It is not yet clear which of the three data analys is methods will be most effective when the targets are buried in salt. The method involving the use of selected reference waveforms to calculate correlation coefficients is particularly attractive because it offers the possibility of a completely automatic, computer controlled, data collection and analysis process.

The use of bentonite, presumably in the form of a bentonite/sand mixture, as a filler material in the canister emplacement holes will introduce the problems of refracted signal paths and potentially severe signal attenuation at near-vertical radar viewing angles. The refraction problem would significantly increase the complexity of the data analysis process due to the necessity of determining the attitude of each refractive interface and calculating appropriate correction terms. A thick layer of bentonite (say $50 \mathrm{~cm}$ or greater) would severely attenuate radar signals transmitted within the circumference of the emplacenent holes.

On the other hand, the problems associated with the use of bentonite may not be significant if satisfactory results can be obtained by utilizing only signals collected at oblique radar viewing angles (i.e., signals collected with the radar antenna outside the circumference of the emplacement hole). The feasibility of this approach is suggested by the success of the Fourier and waveform correlation methods of data analysis as described in Sections 2.2.2 and 2.2.3.

A brine-forming concentration of water in the salt surrounding a canister would represent a worst case situation from the point of view of radar monitoring. Fortunately, the rate of thermally induced moisture accumulation near a hot canister is expected to be only $200 \mathrm{ml}$ per year for the first 10 years after canister burial and somewhat slower thereafter. Apparently, it is not yet clear where this moisture will accumulate or whether it will do so at a concentration sufficient to form brine. If this small amount of moisture were to be absorbed by a bentonite blanket around a canister, it would not have sufficient volume to increase the signal absorption rate to a level where it would hinder the radar monitoring technique. 
A GPR unit for routine canister monitoring operations could be assembled almost entirely from commercially available hardware components. The principal exception is the radar antenna which may require a development effort. The goal of this effort would be to construct a compact, wide-bandwidth, low-distortion antenna with a broad beamwidth. An appropriate basic design would probably be a ridged horn or a constant-angle wedge TEM antenna. A microcomputer-based digital data acquisition unit similar to that used in the experimental part of this study could be interfaced to the radar unit to comprise a complete, sensing system. A highly automated operational mode could be achieved by programming the microcomputer to control the movement of the radar antenna on a measurement plane defined by movable guide rails.

\subsection{RECOMMENDATIONS}

The results of this initial investigation are strongly positive with respect to the feasibility of measuring canister location and orientation by means of a radar backscattering technique. The potential difficulties that are discussed above are speculative and cannot be fully understood or resolved without additional experimental data. Therefore, we recommend that the second phase of this project be initiated.

The work planned to be performed under Phase 2 is focused on experimental measurements at the WIPP facility. The objectives of these measurements and the associated data analysis effort would be to:

1) investigate the propagation of a high-frequency radar signal in the natural salt medium,

2) confirm that useful backscattered radar signals can be obtained from canisters contained in the salt,

3) assess the effects of bentonite backfill on the propagation and backscattering of radar signals,

4) evaluate the performance of at least one alternative radar antenna or system in addition to the GSSI unit, and

5) test and refine data analysis procedures.

Preparations for this experimental effort would involve the burial of several waste canisters in the floor of an experimental room in the underground WIPP facility. Our tentative recommendation is that five canisters be buried. Three of these should be emplaced at a tilt angle of a few degrees. One vertical hole and one tilted hole should be partially or completely backfilled with bentonite (the amount of bentonite would be changed during the test period).

An empty test hole approximately 15 inches in diameter should also be provided. A spherical aluminum radar target lowered into this hole to known depths would provide a means of measuring both the signal velocity and the attenuation rate as a function of depth in the salt. These quantities would be estimated from the travel times and amplitudes of the backscattered signals 
obtained at several slant angles as the spherical target is lowered to progressively greater depths in the test hole.

The radar measurements would be made by manualiy moving the radar antenna across a two-dimensional grid with the aid of guide rails. The position of the antenna would be automatically encoded and recorded along with the radar signals. The digital data recording system described in Section 2.1 would be used to digitize and record the radar data.

The backscattered data from the buried canisters would provide a good estimate of the accuracy to which the location and orientation of the pintle structure of a given canister can be determined when the canister is in place with salt and bentonite backfill. These measurements would also determine the feasibility and accuracy of locating the lower end of a canister. An accurate determiration of the locations of the top and bottom ends of a canister would provide a relatively direct means of measuring canister tilt.

Initial test measurements would be made with the GSSI radar unit. A second set of measurements would be made with an alternative GPR unit utilizing a different type of antenna and a different frequency range. This approach would allow us to verify our understanding of the effects of varying radar system farameters. The commercial unit built by Penetradar (see Section 3) seems particularly suitable for this purpose. Specific radar system parameters to be evaluated include:

Frequency--To what extent does increased attenuation, scattering, and refraction in the salt formation affect the potential gain in resolution thet would be expected at higher frequencies?

Antenna beamwidth--As suggested in Section 3, a broad antenna beamwidth is expected to be needed to provide reasonably uniform illumination of the buried canisters over a wide range of viewing angles. A possible alternative approach would be to use a narrow beam together with a mechanism for aiming the antenna in the proper direction. This approach would probably yield an improved signal-to-noise ratio but would require a more complex monitoring system.

Antenna polarization--In theory, the optimum procedure for identifying the axis of a symmetrical object such as a waste canister involves the use of orthogonally polarized transmitting and receiving antennas. Because a suitable dual-polarization GPR antenna would have to be constructed for this project, it may not be feasible to test this approach under the planned Phase 2 effort.

The data processing methods used in Phase 1 should be refined and extended to three dimensions so that the azimuth angle of canister tilt can be determined. Also, as suggested above, the possibility of calculating canister orientation by using the measured coordinates of both the top and bottom ends of the canister should be investigated. All available data processing methods should be compared using the following criteria: 
- accuracy;

- insensitivity to backfill material and to variations in the salt; and

- suitability for automatic data analysis.

An efficient computer technique for analysis of the radar backscattering data is an important design goal because of the large volume of data that will be produced by an operational canister monitoring system. Operation of the system should require a minimum of operator intervention once the sensing system is installed in a canister storage room and certain basic parameters are entered into the data acquisition unit. 


\section{REFERENCES}

1. J. C. Cook. 1975. "Radar Transparencies of Mine and Tunnel Rocks." Geophysics 40(5): 865-885.

2. C. W. Cook. 1980. "Impulse Radar Scanning of Intact Salt of the Avery Island Mine." SAND80-0632. Sandia National Laboratories, Albuquerque, New Mexico.

3. C. W. Cook. 1982. "Underground Scanning with an Impulse Radar.". SAND 82-0470. Sandia National Laboratories, Albuquerque, New Mexico.

4. R. D. Stewart and R. R. Unterberger. 1976. "Seeing Through Rock Salt With Radar." Geophysics 41(1): 123-132.

5. R. R. Unterberger. 1974. "Electromagnetic Wave Propagation in Rock Salt." In Proceedings, 4th Symposium on Salt, N. Ohio Geo. Soc., Cleveland, Ohio.

6. L. C. Sher, W. C. Savre, J. M. Rice, and R. Athvale. 1985. "Dielectric Properties, Reservoir Rocks at Ultra-High Frequencies." Geophysics $50(4): 692-704$.

7. A. R. Hippel. 1954. Dielectric Materials and Applications. John Wiley \& Sons, Inc., New York, p. 314.

8. J. R. Birchak, C. G. Gardner, J. E. Hipp, and J. M. Victor. 1974. "High Diellectric Constant Microwave Probes for Sensing Soil Moisture." Proceedings of the IEEE 62(1): 93-98.

9. J. R. Lundien. 1971. "Terrain Analysis by Electromagnetic Means, Report 5." U.S. Army Engineer Waterways Experiment Station, Technical Report No. 3-693.

10. R. W. P. King and G. S. Smith. 1981. Antennas in Matter. The MIT Press, Cambridge, Massachusetts, Chapter 6. 
.

. 


\section{BIBLIOGRAPHY}

Annan, A. P., and J. L. Davis. 1977. "Radar Range Analysis for Geological Materials." Report of Activities, Part B, Geological Survey of Canada, Paper 77-1B:117-124.

Arcone, S. A. 1981. "Distortion of Model Subsurface Radar Pulses in Complex Dielectrics." Radio Science, 16(5):855-864.

Arkind, K. 1976. "Realistic Elint Design Considers Antenna Pattern TradeOffs." Microwave Syst. News, 6(5):92-93, 95-97.

Aydin, K., A. ATtintas and A. Hizal. 1983. "Null Field Formulations for Dielectric-Coated Antennas." Radio Science, 18:1225-1242.

Bennett, C. L., and G. F. Ross. 1978. "Time-Domain Electromagnetics and Its Applications." Proceedings of the IEEE, 66:299-318.

Blanco Bautista, J. M. 1984. "Protecting Radar Receivers." Mundo Electron. (Spain), 135:131-135.

Boulanger, R. J., N. 1. Mostowy, A. Mohsen and M. Hamid. 1970. "Radiation Characteristics of Dielectric-Loaded Horn Antennas." Electron. Lett., $\underline{6}: 20-21$.

Brain, D. B., and A. W. Rudoe. 1984. "Efficient Satellite Antennas." Electron. \& Power (GB), 3.

Braun, E. H. 1956. "Some Data for the nesign of Electromagnetic Horns." IRE Trans. Antennas Propan., 4:?9-31.

Bystrom, A., Jr., and D. G. Berntsen. 1956. "An Experimental Investigation of Cavity-Mounted Helical Antennas." IRE Transactions on Antennas and Propagation, January, pp. 53-58.

Caldecott, R., and A. J. Terzuoli, ir. 1979. "An Underground Mapping System Usino Impulse Radar." Seventh IEEE/PES Transmission and Distribution Conference and Exposition, pp. 99-107, April 1-6, 1979, Atlanta, Georoia.

Chandra Mouly, M. C. 1983. "A Pyramidal Horn Antenna with Identical E- and H-Plane Patterns." J. Inst. Eno. (India) Electron.\& Telecommun. Eng. Div., $63: 81-83$.

Changlu, L. 1983. "Short Backfire Antenna Research." International Symposium Digest, Antennas and Propagation, 1:138-141.

Changlu, L., and R. Yingzhen. 19B3. "Analyses of Dipole Antenna with Feedpoint Displacements." Internatinnal Symposium Digest, Antennas and Propagation, $\underline{1}: 145-148$. 
Chaudhuri, S. K., and Y. L. Chow. 1981. "A Baseband Radar with Log-Periodic Antenna and Post-Reception Processing Scheme." Arch. Elektron. and Uebertragungstech. (Germany), 35(10):391-396.

Chew, W. 1982. "A Broad-Band Annular-Ring Microstrip Antenna." IEEE Transactions on Antennas and Propagation, AP-30:918-922.

Cook, J. C. 1960. "Proposed Monocycle-Pulse VHF Radar for Airborne Ice and Snow Measurement." AIEE Communication and Electronics, 51:588-594.

Curtis, 4. L. 1960. "Spiral Antennas." IRE Transactions on Antennas and Propagation, pp. 298-306.

Daniels, D. J. 1977. "The Use of Radar in Geophysical Prospecting." Plessey Padar Research Centre, Havant, Hampshire.

Darracott, B. W., and M. I. Lake. 1981. "Initial Appraisal of Ground Probing Padar for Site Investigation in Britain." Ground Eng., 14(3):14-18.

David, R. A., C. W. Cook and S. D. Stearns. "Array Processing Techniques Applied to Ground Peretration Radar Data." 16th Asilomar Conference on Circuits, Systems, and Computers, November 8-10, 1982.

Dolphin, L. T., R. L. Bollen, D. A. Johnson, G. N. Oetzel and J. D. Tanzi. 1975. "Electromanetic Sounder Experimerts at the Pyramids of Giza." Final Report, Proiect 7663 , Stanford Pesearch Institute.

Dolphir, L. T., Ir., P. I. Bollen and G. N. Detzel. 1974. "An Underaround Flectromagnetic Sounder Experiment." Gecphvsics, 39( ] !: $49-55$.

Cubda1, P. B. 1977. "Horn Antenna Sidelobe Reduction Using Absorber Tunne1s." AP-S International Symposium 1977, pp. 3?4-327, ?une 20-22, 1977, Stanford, California.

Everle, J. W., C. A. Levis and D. McCoy. 1960. "The FIared Siot: A Moderately Directive Flush-Mounted Broad-Band Antenna." IRE Transactions on Antennas and Proparation, September, pp. 461-468.

Elachi, C., T. Bicknell, R. L. Jordan and C. H'U. 1982. "Spaceborne SyntheticAperture Imaging Radars: Applications, Techniques and Technology." Proceedings of the IEEE, 70(10):1174-1209.

Electromagnetics Inst., Tech. Univ. of Denmark. 1979. IEEE Trans. Antennas and Propag., AP-27 (5):640-646.

Evans, S., and F. N. Kong. 1983. "TEM Horn Antenna: Input RefTection Characteristics in Transmission." IEE Proceedings, $130(\mathrm{H})(6): 403-409$.

Evans, J. R., T. E. Fortmann and A. Cantoni. 1977. "Envelope-Constrained Filters: I. Theory and Applications." IEEE Trans. Inf. Theory, IT $-23(4): 421-434$. 
Finkel'shtein, M. I. 1977. "Subsurface Radar." Teleconmun. Radio Eng., $31-32(11): 18-26$.

Franceschetti, G. 1974. "Pulsed Antennas." IEEE Transactions on Antennas and Propagation, AP-22:651-661.

Glotov, V. P., V. A. Kutev, and M. I. Finkel'shtein. 1981. "Realization of Subsurface Sounding of Earth Layers with the Help of a Coherent Radar with Doppler Filtering." Radio Eng. Electron. Phys., 25(3):103-110.

Gogorodskii, V. V., and G. V. Trepov. "Radar Measurements of the Thickness of Peat and Sapropel Beds." Ah. Tekh. Fiz. (USSR), 49(3):670-672.

Griffin, J. M., and J.R. Forrest. 1982. "Rroadband Circular Disc Microstrip Antenna." Electron. Lett. (GR), 18(6):266-269.

Hamid, M., and A. Al-Sulaiman. 1983. "New Types of Dielectric-Loaded Horn Antennas." Int. J.Electron. (GB), 55(5):729-750.

Horton, K. A., R. M. Morey, R. H. Beers, V. Jordan, S. S. Sandler and L. Isaacson. 1981. "An Evaluation of Ground Penetrating Radar for Assessment of Low Level Nuclear Waste Disposal Sites." Report to U.S. Nuclear Regulatory Conmis is inn, NUREG/CR-22I.2.

IEEE. 1980. 1980 International Symposium Digest. Antennas and Propagation.

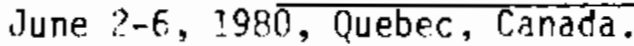

IEEE. 1979. 1970 International Symposium Digest. Antennas and Propagation. June 18-22, 1979, Seattle, Washington.

Isabe11, D. F. 1960. "Log Periodic Dipole Arrays." IRE Transactions on Antennas and Propagation, Mav, pp. 260-267.

James, I. R., and M. B. Amin. 1981. "Techniques for Utilization of Hexagonal Ferrites in Radar Absorbers: II. Reduction of Radar Cross-Section of $H F$ and VHF Wire Antennas." Radio and Electron. Eng. (GB), 51(5):219-225.

Jasik, H., ed. 1961. Antenna Engineering Handbook. McGraw-Hill Bnnk Company, New York.

Jeffrey, Z. 1983. "Whole and Antenna Design Concepts." IREECON International Sydney 83. 19th International Electronics Convention and Exhibition, Digest of Papers, pp. 206-206, September 5-9, 1983, Sydnev, Australia.

Kaiser, J. A. 1960. "The Archimedean Two-Wire Spiral Antenna." IRE Trans., AP-8: $312-373$.

Kaloshin, V. A., and A. A. Eydus. 1983. "On the Radiation of Horn Antennas." Radio Eng. Electron. Phys., 28(1):16-20.

Kanda, M. 1982. "The Effects of Resistive Loading nf "TEM" Horns." IEEE Transactions on Electromagnetic Compatibility, EMC-24(2):245-255. 
Kama?, A. K., and N. K. Agarwal. 1975. "Gain of Surface Wave Horn Antenna." IEEE Trans. Antennas Propag., 23:739-740.

Kao, P. S. 1982. Automated Step-Frequency Radar Cross-Section Measurements." 1982 APS International Symposium Digest, Antennas and Propagation, 1:237-240.

Kellogg, W. C. 1982. "Digital Processing Rescues Hardware Phase Errors (in Radar Systems)." Microwaves and RF (USA), 21(12):63-66, 80.

Kimura, H., C. T. Tai and V. V. Liepa. 1983. "A Study of Spherical Cap Antennas." International Symposium Digest, Antennas and Propagation, 1: $126-129$.

Kong, F. N., and S. Evans. "Microwave Imaging Using an Impulse Signal."

Kraus, ․ D. 1950. Antennas. McGraw-Hill Book Company, New York.

Kumazawa, H., T. Katagi and T. Ebisui. 1983. "Shaped-Beam Horn-Reflector Antennas with Aperture Phase Distribution Modified by a Plane Wave." International Symposium Digest, Antennas and Propagation, 1:338-341.

Lee, K. F. 1984. Principles of Antenna Theory. ISBN: 0471-90167-9 (John Wiley \& Sons, Inc., New York), $324 \mathrm{pp}$.

Lee, K. F., P.-W. Wong and K.-F. Larm. 1982. "Theory of the Frequency Responses of Uniform and Quasi-Taper Helical Antennas." IEEE Transactions on Antennas and Propagation, AP-30:1017-1021.

Lewis, P. 1984. "A Synthetic Aperture Receiver with Holooraphic Recordina." Radio \& Electron. Eng. (GB), 54(1):23-32.

Luchininov, V. S. 1980. "Subsurface Radar Sounding of Stratified Media: Equation of the Sounding Beam." Radiotekh. and Elektron. (USSR), 25(7):1434-1437.

McIntosh, R. E. 1982. "Bounds on the 0ptimum Performance of Planar Antennas for Pulse Radiation." IEEE Transactions on Antennas and Propagation, AP-30(3): 381-389.

McLendon, R., and C. Turner. 1983. "Broadband Sensors for Lethal Defense Suppression." Microwave J., 26(9):85-102.

Mizuasawa, M., S. Urasaki, S.-I. Betsudan and M. Iimori, 1978. "A Dual Doubly Curved Reflector Antenna Havina Gnod Circular Polarization Characteristics." IEEE Trans. Antennas and Propag., AP-26(3):455-458.

Menendez, R. C, and S.-W. Lee. 1982. "Analysis of Rectangular Horn Antennas via Uniform Asymptotic Theory." IEEE Transactions on Antennas and Propagation, $A P-30: 241-250$. 
Mohanar, P., P. A. Pravinkumar and K. G. Nair. 1982. "Axially Symmetric Radiation Patterns from Flanged E-Plane Sectoral Horn Feeds." Indian J. Radio and Space Phys., 11(3):112-115.

Morev, F. M. 1974. "Continuous Subsurface Profiling by Impulse Radar." Proceedings of the Enqineering Foundation Conference on Subsurface Exploration for Underground Excavation and Heavy Construction, Henniker, New Mampshire.

Morey, R. M., and W. S. Harrington, Jr. 1972. "Feasibility Study of Electromagnetic Subsurface Profiling." EPA-R2-72-082, U.S. Environmental Protection Agency, Washington, D.C.

Morris, G. 1982. "A Broad-Band Constant Beamwidth Corrugated Rectangular Horn." IEEE Transactions on Antennas and Propagation, AP-30:966-974.

Nair, K. G., G. P. Srivastava and S. Hariharan. 1969. "Sharpening of E-Plane Radiation Patterns of E-Plane Sectoral Horns by Metallic Grills." IEEE Iransactions on Antennas and Propagation, January, pp. 91-93.

Nakano, H., T. Yamane and 3. Yamauchi. 1983. "Directive Properties of Parasitic Helix and its Application to Circularly Polarized Antenna." IEEE Proc. H (GB), 130(6):391-396.

Nakano, H., J. Yamauchi and K. Noqami. 1983. "Effects of Wire Radius and Arm Bend on a Rectanqular Spiral Antenna." Electron. Lett. (GB), 19(23):957-958.

Nakano, H., K. Hirose and J. Yamauchi. 1984. "Effects of Arm Bend on OneWavelength Dipole Antenna with Asvmmetric Feeding." Int. 1. Electron. (GR), $56(1): 121-125$.

Olver, A. D., P. Yana Kezhong and P. J. B. Clarricoats. 1983. "Dual-Depth Corrugated Horn Design." Conference Proceedings of the 13th European Microwave Conference, pp. 879-884, September 5-8, 1983, Nuremberq, Germany.

Porcello, L. J., et al. 1974. "The Apollo Lunar Sounder Radar System." Proc. IEEE, 62(6):760-783.

Robinson, L. A., W. B. Weir and L. Young. 1974. "Location and Recognition of Discontinuities in Dielectric Media Using Synthetic RF Pulses." Proc. IEEE, $\underline{62}(1): 36-44$.

Sabhnani, N. H., and B. V. Rao. 1984. "Analysis of Microstrip Dipole Antenna." Electron. Lett. (GB), 20(3):116-118.

Schelkunnff, S. A. 1941. "Theory of Antennas of Arbitrary Size and Shape." Proc. I.R.E., 29:493-521.

Shapira, J. 1983. "Simple Pepresentations of Antenna Spatial Radiation Patterns." International Symposium Digest, Antennas and Propagation, 1:112-115.

Shubert, K. A., A. D. Young, and D. L. Moffatt. 1977. "Synthetic Radar Imagery." IEEE Trans. Antennas and Propag., A.P-25(4):477-483. 
Smith, G. S., and L. N. An. 1983. "Loop Antennas for Directive Transmission into a Material Half Space." Radio Sci., 18(5):664-674.

Stutzman, W. L., and G. A. Thiele. 1981. Antenna Theory and Design. John Wiley \& Sons, New York.

Suhler, S. A., W. R. Peters, B. M. Duff and T. E. Owen. 1980. "GroundPenetrating Electromagnetic System." Final Technical Report and Instruction Manua?, U.S. Army, MERADCOM, Fort BeTvoir, VA Contract No. DAAK70-78-C-0149, Southwest Research Institute Project No. 14-5421.

Theodorou, E. A., M. R. Gorman, P. R. Rigg and F. N. Kong. 1981. "8roadband Pulse-0ptimized Antenna." IEEE Proc. H (GB), I28(3):124-130.

Third International Conference on Antennas and Propagation. 1983. Third International Conference on Antennas and Propagation ICAP 83, 2 Vol. (XXVI+533+XXVI+328), April 12-15, 1983, Norwich, England.

Tranquilla, I. M., and G. B. Graham. 1983. "Swept-Frequency Radiation Pattern Anomalies on Helical Antennas." Can. Electr. Eng. J. (Canada), $8(4): 153-156$.

Uenn, K., and N. Osumi. 1983. "Puise-Radar Holography for Underground Object Imaging." International Sympnsium Digest, Antennas and Propagation, pp. $651-654$.

University of Manitoba, Winnipeg, Canada, and Naval Pnstaraduate School, Monterey, California. 1983. "New Types of Dielectric-Loaded Horn Antennas." International Inurnal of Electronics, $5 \underline{5}(5): 720-750$.

Vokurka, V. ?. 1979. "Elliptical Corrugated Horn for Broadcastino Satellite Antennas." Electron. Lett. (GB), 15(20):652-654.

Wacker, P. F. 1982. "linified Theory of Near-Field Analysis and Measurements: Nonmathematical Discussion." IEEE Trans. Antennas and Propagation, AP-30( I):99-107.

Weeks, W. L. 1968. Antenna Engineering. McGraw-Hill Rook Company, New York.

Williams, J. A., S. Kozak and T. J. Rodenbaugh. 1983. "Leak Location Methods for HV Underground Cables." IEEE Trans. Power Appar. and Svst., PAS-102(7):2029-2037.

Wohlers, R. J. "The GWIA, An Extremely Wide Bandwidth Low-Dispersion Antenna." Cornell Aeronautical Laboratory, Inc., Buffaln, New York.

Yamauchi, 1., H. Nakano and S. Hashimoto. 1983. "Polarization Diversity of 4-Arm Archimedean Spiral Antenna." International Symposium Digest, Antennas and Propagation, 1:130-133.

Young, J. D. 1980. "Antenna Development for Pipe Locating Radar Systems." Ohio State University, Columbus, Ohio. 
APPENDIX

RADAR SIGNAL PROPAGATION IN THE GROUND 


\section{APPENDIX}

\section{RADAR SIGNAL PROPAGATION IN THE GROUND}

This brief review of the principles of radar signal, or electromagnetic wave, propagation in the ground is intended to provide a foundation for the discussion presented in the main body of this report. Specifically, this review focuses on identifying the factors that determine the propagation velocity, attenuation rate, and reflection coefficient for a radar signal in a dissipative (slightly conductive) dielectric medium. The soil layers of the earth are a good example of such a medium.

\section{BASIC EQUATIONS}

The propagation of a plane electromagnetic wave in the earth may be described by the expressions

$$
\begin{aligned}
& \vec{E}(z, t)=\vec{E}_{0} e^{i(\omega t-\beta z)} e^{-\alpha z} \\
& \vec{H}(z, t)=\vec{H}_{0} e^{i(\omega t-\beta z)} e^{-\alpha z}
\end{aligned}
$$

where the direction of propagation is taken to be along the $z$ axis; $\vec{E}$ and $\vec{H}$ are, respectively, the electric and magnetic field vectors of the electromagnetic wave; and $\omega=2 \pi$ times the frequency of the radar wave. The terms $\alpha$ and $B$ are functions of the electrical properties of the ground; specifically, the electrical permitivity $\varepsilon$, the electrical conductivity $\sigma$, and the magnetic permeability $\mu$. These parameters, in turn, are complex functions of frequency, temperature, and the composition and texture of the soil.

\section{ELECTRICAL PERMITIVITY}

When an electric field $\vec{E}$ is applied to dielectric material, positive charges within the material tend to move slightly in the direction of the field, while the corresponding negative charges tend to move in the opposite direction. The resulting polarization, $\vec{P}$, (electric dipole moment per unit volume) is proportional to the applied field; that is, $\vec{p}=(\varepsilon-1) \vec{E}$, where is the electrical permitivity of the material. It is standard practice to refer to the permitivity in terms of its value relative to the permitivity of free space. Representing the latter quantity as $\varepsilon_{0}\left(=8.854 \times 10^{-12} \mathrm{farad} / \mathrm{m}\right)$, we define the term $\varepsilon_{r}=\varepsilon / \varepsilon_{0}$, which is referred to as the relative dielectric constant of the material.

Virtually all soils contain some free water in the spaces between the grains of solid material as well as loosely bound water on the surfaces of the grains. In terms of its effect on the permitivity or dielectric constant of the soil, this water is the dominant component. There are two reasons for this. First, there is an energy loss in the material caused by viscosity or 
internal friction which causes the polarization and relaxation of the dipolar water molecules to lag behind the applied electric field at high frequencies. To account for this phase lag and the corresponding energy dissipation in the form of heat, it is customary to write $\varepsilon_{r}$ as a complex number of the form $\varepsilon_{r}=\varepsilon_{r}^{\prime}-i \varepsilon_{r}^{\prime \prime}$. Figure A. 1 illustrates the dependence of $\varepsilon_{r}$ on frequency and temperature for one type of soil.

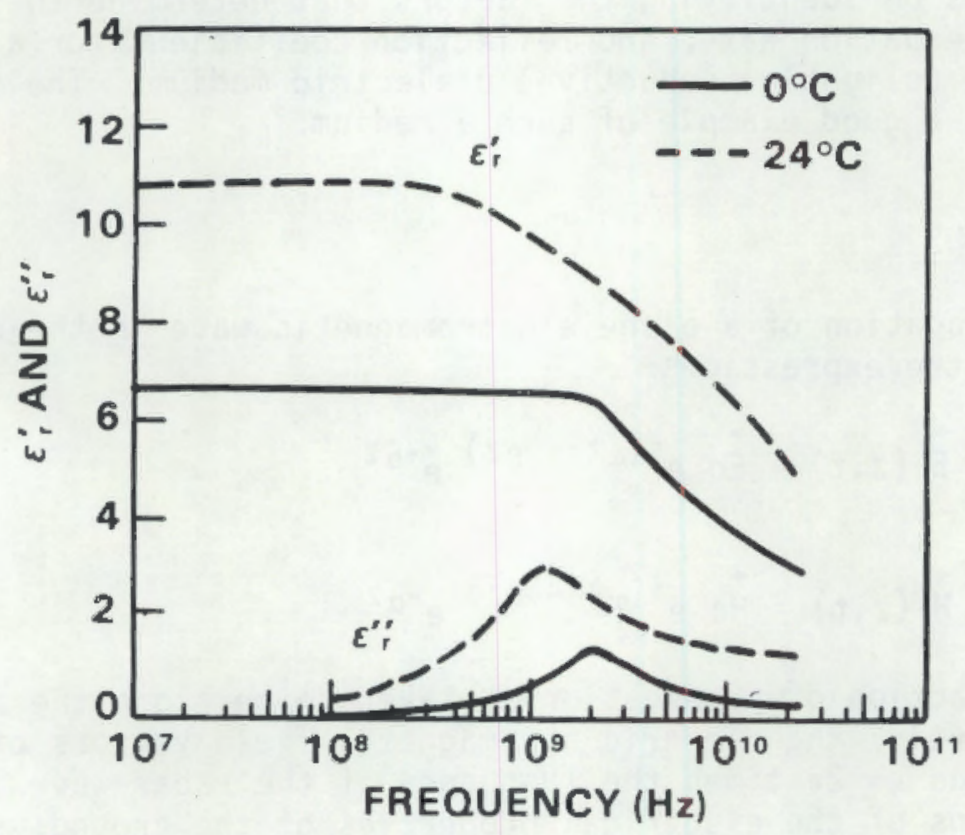

FIGURE A.1. The measured complex dielectric constant of a clay soil with a moisture content of $10 \%$ by weight.

The second reason for the dominating effect of water on soil permitivity is that the real part of the dielectric constant of water is approximately 80 , whereas the corresponding values for the solid components of most rocks and soils lie in the range 4-10. Consequently, the real part of the dielectric constant for a soil can usually be estimated closely by the empirical equation

$$
\varepsilon_{r}^{\prime}=3.03+9.3 M_{v}+146.0 M_{v}^{2}-76.7 M_{v}^{3}
$$

which depends only on the fractional volumetric water content Mv. Figure A.2 is a plot of this equation.

\section{ELECTRICAL CONDUCTIVITY}

The value of this parameter for soils is also dominated by the amount of water contained in the material. This is due to the fact that the electrical conductivity of the dry solid components of a soil is several orders of magnitude lower than that of the free and adsorbed water components. The conductivity of the water is dependent on the number of ions that are free to migrate through the fluid under the influence of an applied electric field. 
However, there is no adequate theory available to explain. in detail the processes of electrical conduction in a soil.

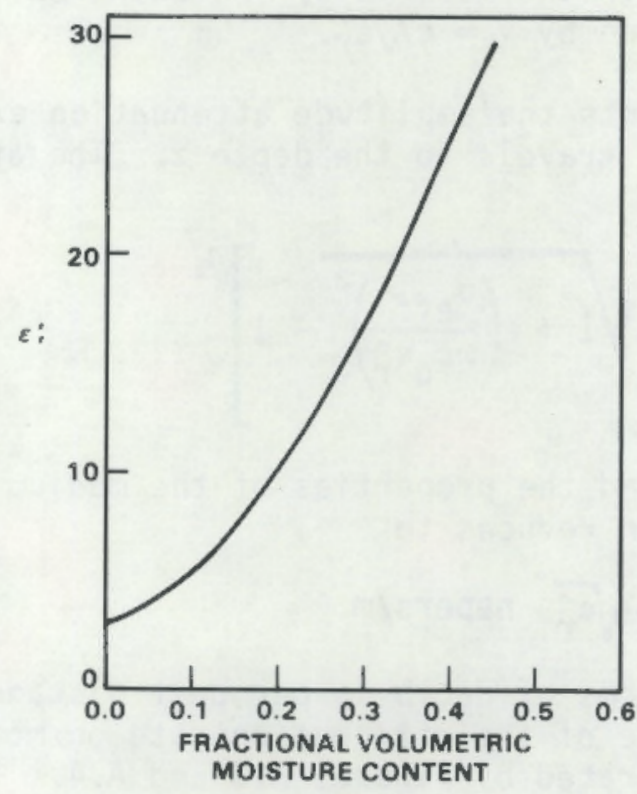

FIGURE A.2. Empirical relationship between $\varepsilon_{r}^{\prime}$ and the volumetric moisture content of mineral soils.

\section{MAGNETIC PERMEABILITY}

The relative magnetic permeability, $\mu_{r}$, is defined as the ratio of the magnetic field in a material to the value that the magnetic field would have in free space; i.e., $\mu_{r}=\mu / \mu_{0}$, where $\mu_{0}$ is the permeability of free space ( $4 \pi$ $x 10^{-7}$ henry/m). The magnitude of $\mu_{r}$ depends on the amount of ferrimagnetic minerals present in the material. In salt, the amount is negligible; thus $\mu_{r}$ $=1$.

\section{SIGNAL VELOCITY AND ATTENUATION}

The term $\left.e^{i(\omega t}-\beta z\right)$ represents a sinusoidal wave traveling in the $z$ direction. If we assume that $\mu_{r}=1$, the propagation velocity of the wave is

$$
v=\frac{\omega}{B}=c \sqrt{\frac{2}{\varepsilon_{r}^{\prime}}}\left[\sqrt{1+\left(\frac{\sigma_{e f f}}{\omega \varepsilon_{0} \varepsilon_{r}^{\prime}}\right)^{2}}+1\right]^{-\frac{1}{2}}
$$

where $c$ is the speed of light in free space. The term $\sigma_{e f f}$ is an effective

\section{A. 3}


electrical conductivity that accounts for both ionic conductivity and dielectric relaxation effects.

For a broad range of ground materials, including salt, the GPR signal velocity can be approximated by $v=c / \sqrt{\varepsilon_{r}^{\prime}}$.

The term $e^{-\alpha z}$ represents the amplitude attenuation experienced by the electromagnetic wave as it travels to the depth $z$. The attenuation constant $a$ is given by

$$
\alpha=\frac{\omega \sqrt{\varepsilon_{r}^{\prime}}}{\sqrt{2} c}\left[\sqrt{1+\left(\frac{\sigma_{\text {eff }}}{\omega \varepsilon_{0} \varepsilon_{r}^{\prime}}\right)^{2}}-1\right]^{\frac{1}{2}}
$$

If the radar frequencies and the properties of the medium are such that ( $\sigma_{e f f} /$ $\left.\omega \varepsilon_{0} \varepsilon_{r}\right)^{2} \ll 1$, this equation reduces to

$$
\alpha \simeq 188 \sigma_{\text {eff }} / \sqrt{\varepsilon_{r}^{\prime}} \text { nepers/m. }
$$

The attenuation rate in terms of decibels per unit distance traveled is $A=$ $8.69 \alpha \mathrm{dB} / \mathrm{m}$. The dependence of the attenuation rate on moisture content and signal frequency is illustrated by Figures A.3 and A.4.

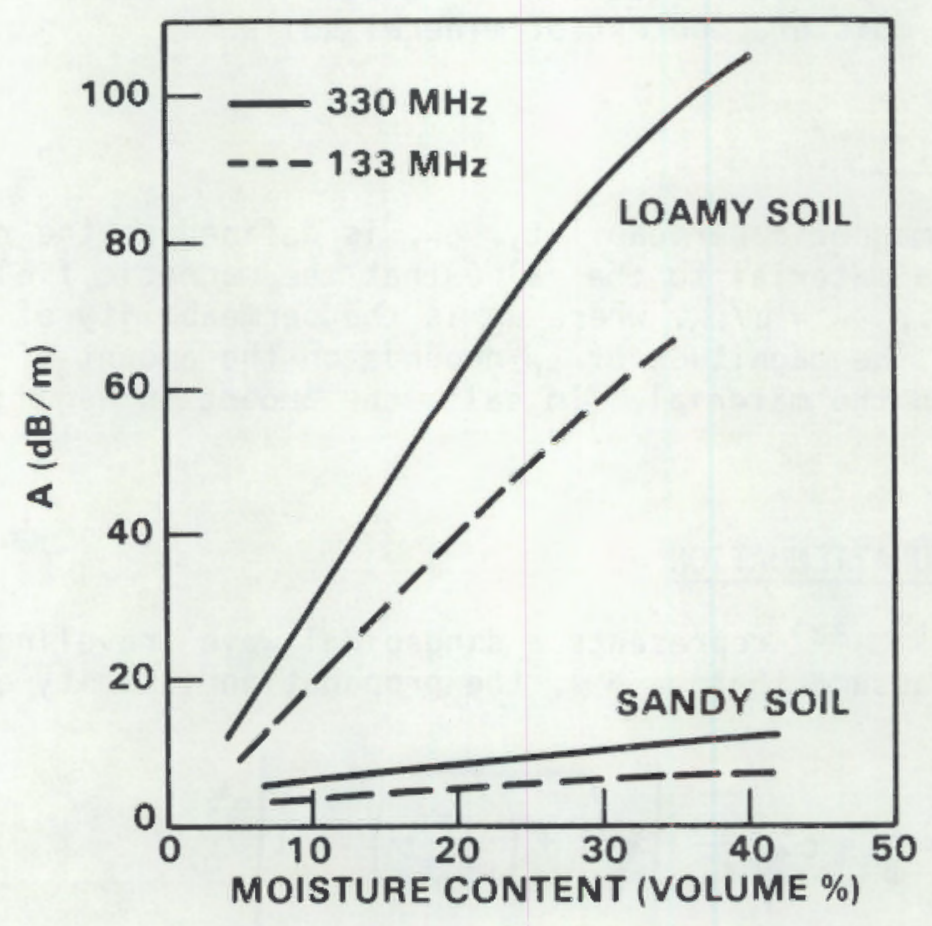

FIGURE A.3. Attenuation rate versus moisture content for two soils and two signal frequencies. 3

$$
\text { A. } 4
$$




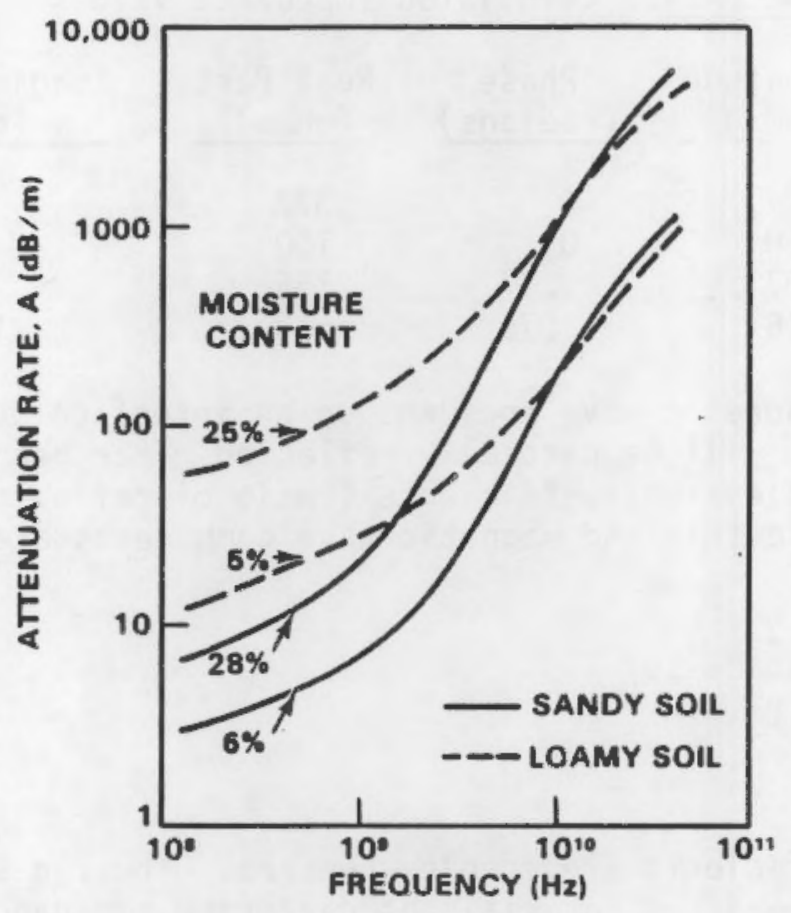

FIGURE A.4. Attenuation rate versus frequency for two soils and two levels of volumetric moisture content. 3

\section{REFLECTION AT INTERFACES}

The ratio of the electric and magnetic components of a transverse electromagnetic wave as it travels through a lossy dielectric material is given by

$$
\frac{E}{H}=\frac{i \mu \omega}{\alpha+i \beta}=\sqrt{\frac{\mu_{0}}{\varepsilon_{0} \varepsilon_{r}}}\left[1+\left(\frac{\sigma_{\text {eff }}}{\frac{\omega \varepsilon_{0} \varepsilon_{r}}{\sigma^{\prime} r}}\right)^{2}\right]^{-\frac{1}{4}} e^{i \phi}
$$

where

$$
\phi=\tan ^{-1}\left(\frac{\alpha}{\beta}\right)
$$

This ratio, which has units of impedance, is defined as the complex intrinsic impedance, $Z$, of the medium. The intrinsic impedance of free space is

$$
z_{0}=\sqrt{\frac{\mu_{0}}{\varepsilon_{0}}}=377 \text { ohms }
$$


a strictly real value. For other media, $Z$ has both real and imaginary components. The following table gives representative impedance values for four materials likely to be significant in the WIPP application.

\section{TABLE A.1. Calculated Impedance Values}

\begin{tabular}{|c|c|c|c|c|}
\hline Material & $\begin{array}{l}\text { Magnitude } \\
\text { (ohms) } \\
\end{array}$ & $\begin{array}{c}\text { Phase } \\
\text { (radians) } \\
\end{array}$ & $\begin{array}{l}\text { Rea 1 Part } \\
\text { (ohms) } \\
\end{array}$ & $\begin{array}{c}\text { Imaginary Part } \\
\text { (ohms) } \\
\end{array}$ \\
\hline $\begin{array}{l}\text { Air } \\
\text { Salt } \\
\text { Bentonite } \\
\text { Salt Water }\end{array}$ & $\begin{array}{r}377 \\
150 \\
157 \\
16\end{array}$ & $\begin{array}{l}0 \\
0 \\
.17 \\
.76\end{array}$ & $\begin{array}{r}377 \\
150 \\
155 \\
12\end{array}$ & $\begin{array}{r}0 \\
0 \\
27 \\
11\end{array}$ \\
\hline
\end{tabular}

A plane electromagnetic wave incident on an interface between two media of differing impedance will be partially reflected. For perpendicular incidence, the complex reflection coefficients (ratio of reflected and incident amplitudes) for the electric and magnetic wave components are

$$
R_{E}=\frac{Z_{2}-Z_{1}}{Z_{2}+z_{1}}=-R_{H}
$$

These reflective coefficients are complex numbers. Thus, a signal reflected from an interface between two materials of differing impedance wi 11 undergo both a reduction in amplitude (the signal will be partially transmitted across the interface) and a shift in phase. 


\section{APPENDIX REFERENCES}

7. P. Hoekstra and A. Delaney. 1974. "Dielectric Properties of Soils at UHF and Microwave Frequencjes." Journal of Geophysical Research 79(11): 1699-1708.

2. G. C. Topp, J. L. Davis, and A. P. Annan. 1980. "Electromagnetic Determination of Soil Water Content: Measurements in Coaxial Transmission Lines." Water Resources Research 16(3): 574-582.

3. Yu I. Leschchanskii, G. N. Lebedova, and V. D. Shumilin. 1971. "The Electrical Parameters of Sandy and Loamy Soils in the Range of Centimeter, Decimeter, and Meter Wavelengths." Radiofjzika 14(4):445-451.

4. A. P. Annan and J. L. Davis, 1977. "Radar Range Analysis for Geologic Materials." Report of Activities, Part B; Geological Survey of Canada, Paper 77-1B: 117-124. 

DISTRIBUTION

No. of

Copies

OFFSI TE

36 Westinghouse Electric Corporation WIPP Project

Carlsbad, NM 88221

30 DOE Technical Information

Center

ONSITE

DOE Richland Operations Office

H. E. Ransom

15 Pacific Northwest Laboratory

G. A. Sandness (5)

K. C. Davis

G. M. Stokes

A. M. Sutey

Publishing Coordination (2)

Technical Information (5) 
